\title{
NORMALITÉ DE CERTAINS ANNEAUX DÉTERMINANTIELS QUANTIQUES
}

\author{
by LAURENT RIGAL
}

(Received 5th January 1998)

\begin{abstract}
Let $\mathbb{K}_{q}[\mathbf{X}]=\mathcal{O}_{q}(M(m, n))$ be the quantization of the ring of regular functions on $m \times n$ matrices and $I_{q}(\mathbf{X})$ be the ideal generated by the $2 \times 2$ quantum minors of the matrix $X=\left(X_{i j}\right)_{1 \leq i \leq m, 1 \leq j \leq n}$ of generators of $\mathbb{K}_{q}[X]$. The residue class ring $R_{q}(\mathbf{X})=\mathbb{K}_{q}[X] / I_{q}(\mathbf{X})$ (a quantum analogue of determinantal rings) is shown to be an integral domain and a maximal order in its division ring of fractions. For the proof we use a general lemma concerning maximal orders that we first establish. This lemma actually applies widely to prime factors of quantum algebras. We also prove that, if the parameter is not a root of unity, all the prime factors of the uniparameter quantum space are maximal orders in their division ring of fractions.
\end{abstract}

1991 Mathematics subject classification: 16D30, 17B37, $16 \mathrm{H} 05$.

\section{Introduction}

Ce travail s'attache à l'étude de certaines algèbres quantiques du point de vue de la normalité et en particulier à celle d'analogues quantiques d'anneaux déterminantiels.

Dans la première section, nous établissons un lemme général concernant la propriété d'ordre maximal (lemme 1.1). Ce lemme est une version non commutative d'un résultat connu en situation commutative (voir [2, lemme 16.24]) sur lequel $\mathbf{M}$. Lazarus a attiré notre attention. Il s'applique très largement aux algèbres quantiques comme nous le verrons dans les sections suivantes. Les résultats sur la notion d'ordre maximal utilisés dans ce travail sont essentiellement dus à $M$. Chamarie et G. Maury (voir [3], [4] et [8]).

Dans la seconde section, nous considérons la quantification $\mathbb{K}_{q}[\mathbf{X}]=\mathcal{O}_{q}(M(m, n))$ de l'anneau des fonctions régulières sur les matrices de format rectangulaire $m \times n$. Nous montrons que, si $I_{q}(\mathbf{X})$ désigne l'idéal de $\mathbb{K}_{q}[\mathbf{X}]$ engendré par les mineurs quantiques de format $2 \times 2$, alors l'anneau quotient $R_{q}(\mathbf{X})=\mathbb{K}_{q}[\mathbf{X}] / I_{q}(\mathbf{X})$ est intègre et un ordre maximal de son corps de fractions.

Ce résultat est donc une quantification de propriétes classiques dans le cas commutatif concernant les anneaux déterminantiels (voir [2]). La preuve développée cidessous est une quantification de celle établie dans le cas commutatif par D. W. Sharpe (voir [15]).

Dans un travail récent (voir [7]), K. R. Goodearl et T. H. Lenagan ont démontré que l'idéal de $\mathcal{O}_{q}(M(m, n))$ engendré par les mineurs quantiques de format $t \times t$ quelconque est complètement premier. 
Dans la troisième section, la même approche est appliquée à l'étude de certains facteurs intègres de l'espace quantique uniparamétré $E_{n}^{q}$. On prouve en particulier que si le corps de base est algébriquement clos et le paramètre $q$ non racine de l'unité, alors tous les facteurs premiers de $E_{n}^{q}$ sont des ordres maximaux de leur corps de fractions.

Notations. Dans tout ce travail, $\mathbb{K}$ désigne un corps commutatif et si $p \in \mathbb{N}^{*}$, on pose $\mathbb{N}_{p}=\{1, \ldots, p\}$. D'autre part, si $R$ est un anneau et si $x$ est un élément de $R$ tel que $\mathcal{X}=\left\{x^{i}, i \in \mathbb{N}\right\}$ soit un ensemble de dénominateurs à droite de $R$, on note $R_{x}$ l'anneau localisé $R \mathcal{X}^{-1}$. De plus, si $R$ est une $\mathbb{K}$-algèbre et $\lambda \in \mathbb{K}^{*}$, pour $(a, b) \in R^{2}$, on pose $[a, b]_{\lambda}=a b-\lambda b a$ (et $\left.[a, b]=a b-b a\right)$. Enfin, la notation $I \triangleleft R$ signifie que $I$ est un idéal de $R$.

\section{Un lemme général sur les ordres maximaux}

Le but essentiel de cette section est de prouver le lemme 1.1. Pour la cohérence de l'ensemble, nous rappelons certains résultats classiques sur les ordres maximaux en suivant [9].

Pour la définition générale d'ordre maximal, nous renvoyons à [9, chap. I]. Nous nous plaçons dans le cadre où $R$ est un anneau noethérien intègre et notons $Q=\operatorname{Frac}(R)$ son corps de fractions. Il est immédiat qu'alors $R$ est un ordre de $Q([9, I \S 2])$. Dans ces conditions, l'anneau $R$ est un ordre maximal de $Q$ si il vérifie la propriété suivante: si $T$ est un anneau tel que $R \subseteq T \subseteq Q$ et tel qu'il existe $a$ et $b$ dans $R \backslash\{0\}$ tels que $a T b \subseteq R$, alors $T=R$.

Si $I$ est un idéal non nul de $R$, on pose $\mathcal{O}_{l}(I)=\{q \in Q \mid q I \subseteq I\}$ et $\mathcal{O}_{r}(I)=$ $\{q \in Q \mid I q \subseteq I\}$. D'après [9, I.3.1], $R$ est un ordre maximal de $Q$ si et seulement si pour tout idéal $I$ non nul de $R, \mathcal{O}_{I}(I)=\mathcal{O}_{r}(I)=R$. Ce critère est utilisé pour la preuve de 1.1 .

Lemme 1.1. Soit $R$ un anneau noethérien intègre et $Q=\operatorname{Frac}(R)$. On suppose qu'il existe un élément normal non nul $x$ de $R$ tel que

(1) $(x)=\bigcap_{i=1}^{r} \mathfrak{p}_{i}$ où, pour $i \in \mathbb{N}_{r}, \mathfrak{p}_{i}$ est un idéal complètement premier de $R$ et

(2) le localisé $R_{x}$ de $R$ en les puissances de $x$ est un ordre maximal de $Q$.

Enfin, on note $\tau$ l'automorphisme associé à $x$ (par $\forall a \in R, a x=x \tau(a)$ ). Dans ces conditions, si pour $i \in \mathbb{N}_{r}, \tau\left(\mathfrak{p}_{i}\right) \subseteq \mathfrak{p}_{i}$ alors $R$ est un ordre maximal de $Q$.

Preuve. Il s'agit de montrer que si $(0) \neq I$ est un idéal de $R$, on a $\mathcal{O}_{l}(I)=\mathcal{O}_{r}(I)=R$.

Soit $(0) \neq I$ un idéal de $R$; montrons que $\mathcal{O}_{l}(I) \subseteq R$ (l'inclusion inverse est évidente). On note $I_{x}$ l'idéal engendré par $I$ dans $R_{x}$, de sorte que $I_{x}=I R_{x}=R_{x} I$ (voir [10, 2.1 .16 (vi)]). Si $q \in \mathcal{O}_{l}(I)$, alors $q I_{x}=q I R_{x} \subseteq I R_{x}=I_{x}$, c'est-à-dire que $q \in \mathcal{O}_{l}\left(I_{x}\right)$. Puisque $R_{x}$ est un ordre maximal, il s'ensuit que $q \in R_{x}$. 
Supposons alors $q \in R_{x} \backslash R$, on a $q=p x^{-s}$, où $s \in \mathbb{N}^{*}$ et $p \in R \backslash(x)$. D'autre part, $q I \subseteq I$ assure que $\forall t \in \mathbb{N}, q^{t} I \subseteq I$. Donc, si $a$ est un élément non nul de $I, \forall t \in \mathbb{N}$, $q^{i} a \in R$. En particulier, le sous- $R$-module à gauche $R[q]$ de $Q$ engendré par les puissances de $q$ est inclus dans le sous- $R$-module à gauche de type fini $R a^{-1}$ de $Q$. $R$ étant noethérien, il s'ensuit que $R[q]$ est noethérien et par conséquent, il existe $n \in \mathbb{N}$ et une famille $\left\{a_{0}, \ldots, a_{n}\right\}$ d'éléments de $R$ tels que $q^{n+1}=a_{n} q^{n}+\ldots+a_{0}$. En posant $\sigma=\tau^{s}$, on obtient que $\left(p x^{-s}\right)^{n+1}=a_{n}\left(p x^{-s}\right)^{n}+\ldots+a_{0}$, puis que $p \sigma(p) \ldots \sigma^{n}(p) x^{-s(n+1)}=$ $a_{n} p \sigma(p) \ldots \sigma^{n-1}(p) x^{-s n}+\ldots+a_{0}$. Il s'ensuit que $p \sigma(p) \ldots \sigma^{n}(p) \in(x)$.

D'autre part, $(x)=\bigcap_{i=1}^{r} p_{i}$, les $p_{i}$ étant des idéaux complètement premiers stables sous l'automorphisme $\tau$ associé à $x$. Il vient que, pour $i \in \mathbb{N}_{r}, p \sigma(p) \ldots \sigma^{n}(p) \in \mathfrak{p}_{i}$. Par suite, tout $\mathfrak{p}_{i}$ contient l'image de $p$ par une certaine puissance de $\tau$ et donc $p$ lui même. Finalement, $p \in \bigcap_{i=1}^{r} \mathfrak{p}_{i}=(x)$, ce qui est une contradiction. Donc $\mathcal{O}_{l}(I) \subseteq R$.

On montre de même que $\mathcal{O}_{r}(I) \subseteq R$.

Remarque 1.2. Nous remercions Marc Chamarie de nous avoir signalé que le lemme 1.1 reste vrai si $R$ et les $\mathfrak{p}_{i}$ sont seulement supposés premiers et que la condition suffisante $\tau\left(\mathfrak{p}_{i}\right) \subseteq \mathfrak{p}_{i}$, pour $i \in \mathbb{N}_{r}$, est aussi nécéssaire (lorsque $\mathfrak{p}_{i}$ est premier minimal au dessus de $(x)$ ).

Remarque 1.3. Soit $R$ un anneau noethérian intègre, ordre maximal de son corps de fractions.

(i) Si $\alpha$ est un automorphisme de $R$ et $\delta$ une $\alpha$-dérivation de $R$, alors $R[x ; \alpha, \delta]$ est un ordre maximal de son corps de fractions ([9, V.2.5]).

(ii) Si $\mathcal{S}$ est un ensemble de dénominateurs à droite et à gauche de $R$, alors $R S^{-1}$ est un ordre maximal de son corps de fractions ([9, IV.2.1]).

\section{L'anneau déterminantiel quantique $R_{q}(\mathrm{X})$}

Cette section s'attache à l'étude de l'anneau déterminantiel quantique $R_{q}(X)$, quotient de $\mathbb{K}_{q}[\mathbf{X}]=\mathcal{O}_{q}(M(m, n))$ par l'idéal $I_{q}(\mathbf{X})$ engendré par les mineurs quantiques $2 \times 2$. Cet anneau est défini dans la première sous-section. Dans la seconde, nous montrons que le quotient du localisé $\mathbb{K}_{q}[\mathbf{X}]_{X_{11}}$ par l'idéal engendré par les mineurs quantiques $2 \times 2$ est un anneau intègre et un ordre maximal de son corps de fractions. Dans la troisième sous-section, nous montrons que $R_{q}(\mathrm{X})$ est un anneau intègre et un ordre maximal de son corps de fractions en relevant les propriétés obtenues pour le localisé $\mathbb{K}_{q}[\mathbf{X}]_{\boldsymbol{X}_{11}}$.

\subsection{Définitions et propriétés élémentaires}

La définition de $\mathcal{O}_{q}(M(m, n))$ rappelée ci-dessous est celle qui figure dans [12] (en changeant $q$ en $q^{-1}$ ) et [11]; nous renvoyons à ces deux articles pour les résultats mentionnés sans preuve. Soient $(m, n) \in \mathbb{N}^{*} \times \mathbb{N}^{*}$ et $q \in \mathbb{K}^{*} ;$ on note $\mathcal{O}_{q}(M(m, n))$ 
l'algèbre engendrée sur $\mathbb{K}$ par $m n$ générateurs notés $X_{i j}$ (ou $X_{i, j}$ si il y a un risque de confusion), $(i, j) \in \mathbb{N}_{m} \times \mathbb{N}_{n}$, soumis aux relations suivantes:

$$
\begin{array}{ll}
X_{i j} X_{i l}=q X_{i l} X_{i j}, & \text { pour } 1 \leq i \leq m, \text { et } 1 \leq j<l \leq n \\
X_{i j} X_{k j}=q X_{k j} X_{i j}, & \text { pour } 1 \leq i<k \leq m, \text { et } 1 \leq j \leq n \\
X_{i j} X_{k l}=X_{k l} X_{i j}, & \text { pour } 1 \leq k<i \leq m, \text { et } 1 \leq j<l \leq n \\
X_{i j} X_{k l}-X_{k l} X_{i j}=\left(q-q^{-1}\right) X_{i l} X_{k j}, & \text { pour } 1 \leq i<k \leq m, \text { et } 1 \leq j<l \leq n
\end{array}
$$

Lorsque $m=n$, on note $\mathcal{O}_{q}(M(n))$ au lieu de $\mathcal{O}_{q}(M(m, n))$.

Il convient de représenter les générateurs de $\mathcal{O}_{q}(M(m, n))$ sous forme matricielle; posons alors $\mathbf{X}=\left(X_{i j}\right)_{1 \leq i \leq m, 1 \leq j \leq n}$. Dans ces conditions, l'algèbre $\mathcal{O}_{q}(M(m, n))$ sera notée $\mathbb{K}_{q}[\mathbf{X}]$.

Il est clair que $\mathbb{K}_{q}[\mathbf{X}]$ est graduée par le degré total en les $X_{i j}$. Pour $d \in \mathbb{N}$, on note $\mathbb{K}_{q}[\mathbf{X}]_{d}$ le sous-espace vectoriel des éléments de degré total $d$. On a alors $\mathbb{K}_{q}[\mathbf{X}]=\bigoplus_{d=0}^{\infty} \mathbb{K}_{q}[\mathbf{X}]_{d}$.

Notations 2.1.1. (1) Lorsque $m=n$, on note $\operatorname{det}_{q}(\mathbf{X})=\sum_{\sigma \in S_{n}}(-q)^{\eta(\sigma)} X_{\sigma(1), 1} \ldots X_{\sigma(n), n}$ le déterminant quantique de $\mathbf{X}\left(S_{n}\right.$ est le groupe des permutations de $\mathbb{N}_{n}$ et $l(\sigma)$ la longueur de $\sigma \in S_{n}$ ). C'est un élément central de $\mathbb{K}_{q}[\mathbf{X}]$ (voir [12, (4.6)]).

(2) Si $\mathbf{Z}$ est une sous-matrice carrée de format $r \times r$ de $\mathbf{X}$, la sous-algèbre de $\mathbb{K}_{q}[\mathbf{X}]$ engendrée par les coefficients de $Z$ est isomorphe à $\mathcal{O}_{q}(M(r))$. Le déterminant quantique de $\mathbf{Z}$ est un élément de $\mathbb{K}_{q}[\mathbf{X}]$. Un tel élément s'appelle un mineur quantique $r \times r$ de $\mathbb{K}_{q}[\mathbf{X}]$.

Ce travail s'intéresse aux mineurs quantiques $2 \times 2$. On pose:

$$
[(i, k) ;(j, l)]_{q}=X_{i j} X_{k l}-q X_{i l} X_{k j}, \text { pour } 1 \leq i<k \leq m \text {, et } 1 \leq j<l \leq n ;
$$

c'est le mineur quantique associé à la matrice extraite de $\mathbf{X}$ en ne conservant que les lignes $i, k$ et les colonnes $j, l$. On note alors $\min _{q}(\mathbf{X})$ l'ensemble des mineurs quantiques $2 \times 2$ de $\mathbb{K}_{q}[\mathbf{X}]$ (si $n=1$ ou $\left.m=1, \min _{q}(\mathbf{X})=\emptyset\right)$. Enfin, $I_{q}(\mathbf{X})$ désigne l'idéal de $\mathbb{K}_{q}[\mathbf{X}]$ engendré par $\min _{q}(\mathbf{X})$ et $R_{q}(\mathbf{X})$ l'anneau quotient associé:

$$
I_{q}(\mathbf{X})=\left(\min _{q}(\mathbf{X})\right) \triangleleft \mathbb{K}_{q}[\mathbf{X}] \text { et } R_{q}(\mathbf{X})=\mathbb{K}_{q}[\mathbf{X}] / I_{q}(\mathbf{X}) .
$$

Remarque 2.1.2. La structure d'anneau gradué de $\mathbb{K}_{q}[X]$ assure que, pour $(i, j) \in \mathbb{N}_{m} \times \mathbb{N}_{n}, X_{i j} \notin I_{q}(\mathbf{X})$. De plus, l'idéal $\left(X_{i j}, 1<i \leq m, 1 \leq j \leq n\right)$ de $\mathbb{K}_{q}[\mathbf{X}]$ est complètement premier, contient $I_{q}(\mathbf{X})$ et ne contient aucun $X_{1 j}$, pour $1 \leq j \leq n$. Il s'ensuit que $I_{q}(\mathbf{X})$ ne contient aucun monôme en les $X_{1 j}$, pour $1 \leq j \leq n$.

Remarque 2.1.3. L'algèbre $\mathcal{O}_{q}(M(2))$ est engendrée par $X_{11}, X_{12}, X_{21}$ et $X_{22}$. On note $\delta$ son déterminant quantique. Compte tenu de la relation $X_{11} X_{22}-X_{22} X_{11}=$ 
$\left(q-q^{-1}\right) X_{12} X_{21}$, il vient $\delta=X_{11} X_{22}-q X_{12} X_{21}=X_{22} X_{11}-q^{-1} X_{12} X_{21}$. On a également $X_{11} X_{22}-q^{2} X_{22} X_{11}=\left(1-q^{2}\right) \delta$. Cette relation assure que $R_{q}(\mathbf{X})$ est isomorphe à un quotient d'espace quantique.

$\mathbb{K}_{q}[\mathbf{X}]$ comme extension de Ore itérée. Si $m \geq 2$ (resp. $n \geq 2$ ), on note $\mathbf{X}^{\prime}$ (resp. $\mathbf{X}^{\prime \prime}$ ) la matrice extraite de $X$ en supprimant la $m$-ème ligne (resp. la $n$-ème colonne): $\mathbf{X}^{\prime}=\left(X_{i j}\right)_{1 \leq i \leq m-1,1 \leq j \leq n}$ et $\mathbf{X}^{\prime \prime}=\left(X_{i j}\right)_{1 \leq i \leq m, 1 \leq j \leq n-1}$.

La sous-algèbre de $\mathbb{K}_{q}[X]$ engendrée par les coefficients de $X^{\prime}$ (resp. $X^{\prime \prime}$ ) est isomorphe à $\mathcal{O}_{q}(M(m-1, n))=\mathbb{K}_{q}\left[\mathbf{X}^{\prime}\right]$ (resp. $\left.\mathcal{O}_{q}(M(m, n-1))=\mathbb{K}_{q}\left[\mathbf{X}^{\prime \prime}\right]\right)$; nous identifierons ces algèbres isomorphes. Nous allons décrire $\mathbb{K}_{q}[\mathbf{X}]$ comme extension de Ore itérée de $\mathbb{K}_{q}\left[\mathbf{X}^{\prime}\right]$ et comme extension de Ore itérée de $\mathbb{K}_{q}\left[\mathbf{X}^{\prime \prime}\right]$. Pour ce faire, on introduit les tableaux suivants, pour $s \in \mathbb{N}_{n}$ :

$$
\mathbf{X}_{(1, \ldots, s)}^{\prime}=\left[\begin{array}{cccccc}
X_{11} & \ldots & X_{1 s} & \ldots & \ldots & X_{1 n} \\
\ldots & \ldots & \ldots & \ldots & \ldots & \ldots \\
\ldots & \ldots & \ldots & \ldots & \ldots & \ldots \\
X_{m-1,1} & \ldots & X_{m-1 . s} & \ldots & \ldots & X_{m-1, n} \\
X_{m 1} & \ldots & X_{m s} & & &
\end{array}\right.
$$

Toutes les notations introduites pour les matrices s'étendent à ces tableaux. En particulier, on note $\mathbb{K}_{q}\left[\mathbf{X}^{\prime}{ }_{11, \ldots, s]}\right]$ l'algèbre engendrée par les coefficients du tableau $\mathbf{X}_{(1, \ldots, s)}^{\prime}$ soumis aux relations définissant $\mathbb{K}_{q}[\mathbf{X}]$ et dans lesquelles n'interviennent que les coefficients figurant dans $\mathbf{X}_{\{1, \ldots, s\}}^{\prime}$. Ainsi, $\mathbb{K}_{q}\left[\mathbf{X}_{(1, \ldots, s\}}^{\prime}\right]$ est isomorphe à la sous-algèbre de $\mathbb{K}_{q}[\mathbf{X}]$ engendrée pas les $X_{i j}$, pour $(i, j) \in \mathbb{N}_{m-1} \times \mathbb{N}_{n}$ et les $X_{m j}$ pour $j \in \mathbb{N}_{s}$. Il est clair alors que pour $i \in \mathbb{N}_{n}$, il existe un automorphisme $\sigma_{m i}$ et une dérivation tordue à gauche $\left(\sigma_{m i}, \delta_{m i}\right)$ tels que, si $s \in \mathbb{N}_{n-1}$ :

$$
\mathbb{K}_{q}\left[\mathbf{X}_{(11)}^{\prime}\right]=\mathbb{K}_{q}\left[\mathbf{X}^{\prime}\right]\left[X_{m 1} ; \sigma_{m 1}, \delta_{m 1}\right] \text { et } \mathbb{K}_{q}\left[\mathbf{X}^{\prime}{ }_{(1, \ldots, s+1]}\right]=\mathbb{K}_{q}\left[\mathbf{X}^{\prime}{ }_{11, \ldots, s]}\right]\left[X_{m, s+1} ; \sigma_{m, s+1}, \delta_{m, s+1}\right] .
$$

Enfin, on définit de façon évidente $\min _{q}\left(\mathbf{X}^{\prime}{ }_{(1, \ldots, s)}\right)$ et $I_{q}\left(\mathbf{X}_{(1, \ldots, s)}^{\prime}\right), s \in \mathbb{N}_{n}$. Naturellement, on peut procéder de la même façon à partir de la matrice $\mathbf{X}^{\prime \prime}$. Finalement:

$$
\begin{aligned}
& \mathbb{K}_{q}[\mathbf{X}]=\mathbb{K}_{q}\left[\mathbf{X}^{\prime}\right]\left[X_{m 1} ; \sigma_{m 1}, \delta_{m 1}\right] \ldots\left[X_{m n} ; \sigma_{m n}, \delta_{m n}\right], \\
& \mathbb{K}_{q}[\mathbf{X}]=\mathbb{K}_{q}\left[\mathbf{X}^{\prime \prime}\right]\left[X_{1 n} ; \sigma_{1 n}, \delta_{1 n}\right] \ldots\left[X_{m n} ; \sigma_{m n}, \delta_{m n}\right]
\end{aligned}
$$

On en déduit, par récurrence, que $\mathbb{K}_{q}[\mathbf{X}]$ est une extension de Ore itérée de $\mathbb{K}$; en particulier c'est une algèbre intègre et noethérienne.

Une localisation de $\mathbb{K}_{q}[\mathbf{X}]$. On note $\mathcal{S}_{11}=\left\{\lambda X_{11}^{i}, i \in \mathbb{N}, \lambda \in \mathbb{K}^{*}\right\}$ la partie multiplicative de $\mathbb{K}_{q}[\mathbf{X}]$ engendrée par $X_{11}$ et $\mathbb{K}^{*}$.

Proposition 2.1.4. La partie multiplicative $\mathcal{S}_{11}$ est un ensemble de dénominateurs à 
droite de $\mathbb{K}_{q}[\mathbf{X}]$; on note $\mathbb{K}_{q}[\mathbf{X}]_{X_{11}}=\mathbb{K}_{q}[\mathbf{X}] \mathcal{S}_{11}^{-1}$, le localisé à droite de $\mathbb{K}_{q}[\mathbf{X}]$ vis-à-vis de $\mathcal{S}_{11}$. De plus, si $m \geq 2$, il existe un isomorphisme d'algèbres

$$
\begin{aligned}
\mathbb{K}_{q}[\mathbf{X}]_{X_{11}} & \rightarrow \mathbb{K}_{q}\left[\mathbf{X}^{\prime}\right]_{X_{11}}\left[X_{m 1} ; \sigma_{m 1}, \delta_{m 1}\right] \ldots\left[X_{m n} ; \sigma_{m n}, \delta_{m n}\right] \\
X_{i j} & \mapsto X_{i j} .
\end{aligned}
$$

Preuve. On fixe $n$ et on procède par récurrence sur $m$. Le résultat est vrai lorsque $m=1$ car dans ce cas $\mathbb{K}_{q}[\mathbf{X}]$ est un espace quantique uniparamétré dans lequel $X_{11}$ est normal. Supposons le résultat acquis jusqu'à l'ordre $m-1$; d'après (1) $\mathbb{K}_{q}[\mathbf{X}]$ est une extension de Ore itérée de $\mathbb{K}_{q}\left[\mathbf{X}^{\prime}\right]$. Les relations de définition de $\mathbb{K}_{q}[\mathbf{X}]$ assurent que $\sigma_{m 1}\left(X_{11}\right)=q^{-1} X_{11}$ et que, pour $2 \leq j \leq n, \sigma_{m j}\left(X_{11}\right)=X_{11}$. On est donc dans les hypothèses de $[6,1.4]$ qui permet de conclure.

Remarque 2.1.5. Comme en 2.1.4, on montre que si $s \in \mathbb{N}_{n}$, alors $\mathcal{S}_{11}$ est un ensemble de dénominateurs à droite de $\mathbb{K}_{q}\left[\mathbf{X}_{\{1, \ldots, s\}}^{\prime}\right]$. On pose $\mathbb{K}_{q}\left[\mathbf{X}_{\{1, \ldots, s\}}^{\prime}\right]_{X_{11}}=\mathbb{K}_{q}\left[\mathbf{X}_{\{1 \ldots, \ldots, j}^{\prime}\right] \mathcal{S}_{11}^{-1} ;$ alors, il existe un isomorphisme $\psi: \mathbb{K}_{q}\left[\mathbf{X}^{\prime}{ }_{11, \ldots, s}\right]_{X_{11}} \rightarrow \mathbb{K}_{q}\left[\mathbf{X}^{\prime}\right]_{X_{11}}\left[X_{m 1} ; \sigma_{m 1}, \delta_{m 1}\right] \ldots\left[X_{m s} ; \sigma_{m s}, \delta_{m s}\right]$ tel que $\psi\left(X_{i j}\right)=X_{i j}$. Dans la suite, on identifiera les anneaux concernés.

Deux isomorphismes des anneaux de matrices quantiques. Les isomorphismes introduits à présent seront utiles dans la suite. Nous ne donnons pas la preuve des deux propositions suivantes car elle est immédiate (lorsque $m=n$, voir $[12,3.7]$ ).

Le premier est un isomorphisme de transposition entre $\mathcal{O}_{q}(M(m, n))$ et $\mathcal{O}_{q}(M(n, m))$. On conserve les notations précédentes et on pose $\tilde{\mathbf{X}}=\left(\tilde{X}_{i j}\right)_{1 \leq i \leq n, 1 \leq j \leq m}$ (matrice des générateurs de $\left.\mathcal{O}_{q}(M(n, m))\right)$, de sorte que $\mathbb{K}_{q}[\tilde{\mathbf{X}}]=\mathcal{O}_{q}(M(n, m))$.

Proposition 2.1.6. Il existe un isomorphisme d'algèbres, $\operatorname{Tr}: \mathbb{K}_{q}[\mathbf{X}] \rightarrow \mathbb{K}_{q}[\tilde{\mathbf{X}}]$, défini par $\operatorname{Tr}\left(X_{i j}\right)=\tilde{X}_{j i}$. De plus, $\operatorname{Tr}$ induit une bijection de $\min _{q}(\mathbf{X})$ sur $\min _{q}(\tilde{\mathbf{X}})$ et $\operatorname{Tr}\left(I_{q}(\mathbf{X})\right)=I_{q}(\tilde{\mathbf{X}})$.

Le second est un isomorphisme de «symétrie» entre $\mathcal{O}_{q}(M(m, n))$ et $\mathcal{O}_{q^{-1}}(M(m, n))$. On note $Y_{i j}$ les générateurs canoniques de $\mathcal{O}_{q^{-1}}(M(m, n))$ et $\mathbf{Y}=\left(Y_{i j}\right)_{1 \leq i \leq m, 1 \leq j \leq n}$. Ainsi, $\mathbb{K}_{q^{-1}}[\mathbf{Y}]=\mathcal{O}_{q^{-1}}(M(m, n))$.

Propostion 2.1.7. Il existe un isomorphisme d'algèbres, $\mathbf{S}: \mathbb{K}_{q}[\mathbf{X}] \rightarrow \mathbb{K}_{q}{ }^{-1}[\mathbf{Y}]$, défini par $\mathbf{S}\left(X_{i j}\right)=Y_{m+1-i, n+1-j}$. De plus, $\mathbf{S}$ induit une bijection de $\min _{q}(\mathbf{X})$ sur $\min _{q-1}(\mathbf{Y})$ et $\mathbf{S}\left(I_{q}(\mathbf{X})\right)=I_{q^{-1}}(\mathbf{Y})$.

Relations entre générateurs et mineurs quantiques $2 \times 2$ dans $\mathcal{O}_{q}(M(3))$. Nous procédons, à présent, à une étude détaillée de $\mathcal{O}_{q}(M(3))$; elle est justifiée par la remarque suivante.

Remarque 2.1.8. Considérons un mineur quantique $2 \times 2[(i, k) ;(j, l)]_{q}$ et un générateur $X_{s t}$ de $\mathbb{K}_{q}[\mathbf{X}]$. Ces deux éléments sont contenus dans la sous-algèbre de $\mathbb{K}_{q}[\mathbf{X}]$ 
engendrée par les coefficients de la matrices extraite de $\mathbf{X}$ en ne conservant que les lignes $i, k, s$ et les colonnes $j, l, t$. Cette sous-algèbre est isomorphe à $\mathcal{O}_{q}(M(2))$, $\mathcal{O}_{q}(M(2,3)), \mathcal{O}_{q}(M(3,2))$ ou $\mathcal{O}_{q}(M(3))$. Ainsi, des relations entre générateurs et mineurs quantiques $2 \times 2$ de $\mathcal{O}_{q}(M(3))$ on peut déduire les relations entre générateurs et mineurs quantiques $2 \times 2$ de $\mathbb{K}_{q}[\mathbf{X}]$.

Nous notons $\Delta$ le déterminant quantique de $\mathcal{O}_{q}(M(3))$. Par ailleurs, comme dans [12], si $(k, l) \in \mathbb{N}_{3}^{2}$, on note $A(k l)$ le déterminant quantique de la matrice extraite de $\left(X_{i j}\right)_{1 \leq i, j \leq 3}$ en supprimant la ligne $k$ et la colonne $l$. Enfin, on pose $C_{k l}=(-q)^{l-k} A(k l)$; c'est le cofacteur de $X_{k l}$. On considère alors la transposée $\left(C_{j i}\right)_{1 \leq i, j \leq 3}$ de la matrice des cofacteurs. En notant $I d_{3}$ la matrice identité de format $3 \times 3$, on a (voir $[12,4.6]$ et $[11,(1.17)])$ :

$$
\left(C_{j i}\right)_{1 \leq i, j \leq 3}\left(X_{i j}\right)_{1 \leq i, j \leq 3}=\Delta . I d_{3}=\left(X_{i j}\right)_{1 \leq i, j \leq 3}\left(C_{j i}\right)_{1 \leq i, j \leq 3} .
$$

Enfin, les relations de commutation suivantes se déduisent de [12, (4.4.4), (4.5.1), (4.6.1) et (5.1.2)].

- $A(31)$ est normal dans $\mathcal{O}_{q}(M(3))$.

$A(31)$ commute avec $X_{12}, X_{13}, X_{22}, X_{23}$ et $X_{31}$,

$\left[A(31), X_{11}\right]_{q^{-1}}=\left[A(31), X_{21}\right]_{q^{-1}}=\left[A(31), X_{32}\right]_{q}=\left[A(31), X_{33}\right]_{q}=0$.

- $A(32)$ est normal modulo $(A(31))$ dans $\mathcal{O}_{q}(M(3))$.

$A(32)$ commute avec $X_{11}, X_{13}, X_{21}, X_{23}$,

$\left[A(32), X_{31}\right]_{q}=\left[A(32), X_{33}\right]_{q}=0,\left[A(32), X_{32}\right]=\left(q-q^{-1}\right) X_{31} A(31)$,

$\left[A(32), X_{12}\right]_{q}{ }^{-1}=\left(1-q^{-2}\right) X_{11} A(31),\left[A(32), X_{22}\right]_{q^{-1}}=\left(1-q^{-2}\right) X_{21} A(31)$.

- $A(33)$ est normal modulo $(A(31), A(32))$ dans $\mathcal{O}_{q}(M(3))$.

$A(33)$ commute avec $X_{11}, X_{12}, X_{21}, X_{22}$,

$\left[A(33), X_{13}\right]_{q}=\left[A(33), X_{23}\right]_{q}=\left[A(33), X_{31}\right]_{q}=\left[A(33), X_{32}\right]_{q}=0$,

$\left[A(33), X_{33}\right]=\left(q-q^{-1}\right)\left(-q^{-1} X_{31} A(31)+X_{32} A(32)\right)$.

- $A(23)$ est normal modulo ( $A(31), A(32), A(33))$ dans $\mathcal{O}_{q}(M(3))$.

$A(23)$ commute avec $X_{11}, X_{12}, X_{31}, X_{32}$,

$\left[A(23), X_{13}\right]_{q}=\left[A(23), X_{33}\right]_{q}=0$,

$\left[A(23), X_{21}\right]_{q}=\left(1-q^{2}\right) X_{31} A(33),\left[A(23), X_{22}\right]_{q}=\left(1-q^{2}\right) X_{32} A(33)$,

$\left[A(23), X_{23}\right]_{q^{2}}=\left(q-q^{-1}\right)\left(X_{31} A(31)-q X_{32} A(32)\right)$.

- $A(22)$ est normal modulo ( $A(31), A(32), A(33), A(23))$ dans $\mathcal{O}_{q}(M(3))$.

$A(22)$ commute avec $X_{11}, X_{31}, X_{13}, X_{33}$,

$\left[A(22), X_{12}\right]_{q}=\left(1-q^{2}\right) X_{13} A(23),\left[A(22), X_{21}\right]_{q}=\left(1-q^{2}\right) X_{31} A(32)$,

$\left[A(22), X_{32}\right]_{q}=\left(1-q^{2}\right) X_{33} A(23),\left[A(22), X_{23}\right]_{q}=\left(1-q^{2}\right) X_{33} A(32)$,

$\left[A(22), X_{22}\right]_{q^{2}}=\left(1-q^{2}\right)\left(X_{33} A(33)+X_{31} A(31)+q X_{23} A(23)\right)$.

Pour établir la dernière identité de (7), on utilise $\left[A(23), X_{23}\right]_{q^{2}}=\left[A(23), X_{23}\right]+$ $\left(1-q^{2}\right) X_{23} A(23)$ et on applique successivement (5.1.2) et (4.4.4) de [12]. La dernière identité de (8) s'obtient à partir de $\left[A(22), X_{22}\right]_{q^{2}}=q^{2}\left[A(22), X_{22}\right]+\left(1-q^{2}\right) A(22) X_{22}$ en 
appliquant là encore (5.1.2) puis (4.4.4) de [12] ainsi que l'expression de [A(32), $\left.X_{32}\right]$ mentionnée dans (5).

\subsection{Sur l'idéal des mineurs quantiques $2 \times 2$ de $\mathbb{K}_{q}[X]_{X_{11}}$}

L'objet de cette sous-section est l'étude de l'idéal $\tilde{I}_{q}(\mathbf{X})$ de $\mathbb{K}_{q}[\mathbf{X}]_{X_{11}}$ défini en 2.2.1. Nous montrons que $\mathbb{K}_{q}[\mathbf{X}]_{X_{11}} / \tilde{I}_{q}(\mathbf{X})$ est intègre et un ordre maximal de son corps de fractions. On reprend les notations et conventions introduites en 2.1.4 et 2.1.5. Rappelons que l'anneau intègre $\mathbb{K}_{q}[\mathbf{X}]$ s'injecte canoniquement dans son localisé $\mathbb{K}_{q}[\mathbf{X}]_{\boldsymbol{X}_{11}}$.

Définition 2.2.1. On note $\tilde{I}_{q}(\mathbf{X})$ l'idéal de $\mathbb{K}_{q}[\mathbf{X}]_{X_{11}}$ engendré par les mineurs quantiques $2 \times 2: \tilde{I}_{q}(\mathbf{X})=\left(\min _{q}(\mathbf{X})\right)=\left([(i, k) ;(j, l)]_{q}, 1 \leq i<k \leq m, 1 \leq j<l \leq n\right)$.

Remarque 2.2.2. (1) D'après $\left[10,2.1 .16\right.$ (vi)], $\tilde{I}_{q}(\mathbf{X})=I_{q}(\mathbf{X}) \mathbb{K}_{q}[\mathbf{X}]_{X_{11}}$. De plus, $\tilde{I}_{q}(\mathbf{X})$ est un idéal propre. En effet, supposons qu'il existe $j \in \mathbb{N}_{n}$ tel que $X_{1 j} \in \tilde{I}_{q}(\mathbf{X})$. Alors, il existe $s \in \mathbb{N}$ tel que $X_{1 j} X_{11}^{s} \in I_{q}(\mathbf{X})$, ce qui contredit 2.1.2. En utilisant l'isomorphisme Tr (prolongé aux localisés en $X_{11}$ ), il vient donc que pour $j \in \mathbb{N}_{n}, X_{1 j} \notin \tilde{I}_{q}(\mathbf{X})$ et pour $i \in \mathbb{N}_{m}, X_{i 1} \notin \tilde{I}_{q}(\mathbf{X})$.

(2) Ce qui précède s'étend aux anneaux $\mathbb{K}_{q}\left[\mathbf{X}_{(11 \ldots, s]}^{\prime}\right]_{X_{11}}, s \in \mathbb{N}_{n}$ (voir $\S 2.1$ ). On note $\tilde{I}_{q}\left(\mathbf{X}_{(1, \ldots, s)}^{\prime}\right)$ l'idéal de $\mathbb{K}_{q}\left[\mathbf{X}_{(1 \ldots, \ldots, s}^{\prime}\right]_{X_{11}}$ engendré par les mineurs quantiques $2 \times 2$ de $\mathbf{X}_{(1, \ldots, s)}^{\prime}$. Comme précédemment, $\tilde{I}_{q}\left(\mathbf{X}_{(1, \ldots, s)}^{\prime}\right)=I_{q}\left(\mathbf{X}_{(1, \ldots, s)}^{\prime}\right) \mathbb{K}_{q}\left[\mathbf{X}_{(1, \ldots, s)}^{\prime}\right]_{X_{11}}$. De plus, $\tilde{I}_{q}\left(\mathbf{X}_{(1, \ldots, s)}^{\prime}\right)$ est un idéal propre car si il contenait $X_{11}, \tilde{I}_{q}(\mathbf{X})$ contiendrait aussi $X_{11}$ ce qui est faux d'après le (1).

Nous commençons par donner une famille de générateurs restreinte de $\tilde{I}_{q}(\mathbf{X})$.

Proposition 2.2.3. $\tilde{I}_{q}(\mathbf{X})$ est engendré par $\min _{q}\left(\mathbf{X}^{\prime}\right)$ et les $[(1, m) ;(1, l)]_{q}, 2 \leq l \leq n$.

Preuve. Posons $\tilde{I}_{q}^{\sharp}(\mathbf{X})=\left(\min _{q}\left(\mathbf{X}^{\prime}\right),[(1, m) ;(1, l)]_{q}, 2 \leq l \leq n\right) \triangleleft \mathbb{K}_{q}[\mathbf{X}]_{X_{11}}$. L'inclusion $\tilde{I}_{q}^{\mathbb{Z}}(\mathbf{X}) \subseteq \tilde{I}_{q}(\mathbf{X})$ est triviale. Pour l'inclusion inverse, il s'agit de montrer que tous les mineurs de la forme $[(i, m) ;(j, l)]_{q},(i, j) \neq(1,1)$, sont dans $\tilde{I}_{q}^{\sharp}(\mathbf{X})$. Soit $[(i, m) ;(j, l)]_{q}$ un tel mineur.

1-er cas: $i=1$ et $j \neq 1$. D'après (3), dans la sous-algèbre de $\mathbb{K}_{q}[\mathbf{X}]$ engendrée par les $X_{r s}$ où $r=1, m$ et $s=1, j, l$ (qui est isomorphe à $\mathcal{O}_{q}(M(2,3))$ ), on a $q^{-2} X_{11}[(1, m) ;(j, l)]_{q}-$ $q^{-1} X_{1 j}[(1, m) ;(1, l)]_{q}+X_{1 l}[(1, m) ;(1, j)]_{q}=0$, d'où $[(1, m) ;(j, l)]_{q} \in \tilde{I}_{q}^{\sharp}(\mathbf{X})$.

2-ème cas: $i \neq 1$ et $j=1$. D'après (3), dans la sous-algèbre de $\mathbb{K}_{q}[\mathbf{X}]$ engendrée par les $X_{r s}$ où $r=1, i, m$ et $s=1, l$ (qui est isomorphe à $\mathcal{O}_{q}(M(3,2))$ ), on a la relation $q^{2}[(i, m) ;(1, l)]_{q} X_{11}-q[(1, m) ;(1, l)]_{q} X_{i 1}+\left[(1, i) ;(1, D]_{q} X_{m 1}=0\right.$. Donc $[(i, m) ;(1, l)]_{q} \in \tilde{I}_{q}^{\sharp}(\mathbf{X})$.

3-ème cas: $i \neq 1$ et $j \neq 1$. Soit $\mathbf{Z}$ la sous-matrice de $\mathbf{X}$ obtenue en ne conservant que les lignes $1, i, m$ et les colonnes $1, j, l$ et notons $[(1, i, m) ;(1, j, l)]_{q}$ le déterminant quantique de $\mathbf{Z}$. La sous-algèbre de $\mathbb{K}_{q}[\mathbf{X}]$ engendrée par les coefficients de $\mathbf{Z}$ étant isomorphe à $\mathcal{O}_{q}(M(3))$, d'après (3): 


$$
\begin{aligned}
{[(1, i, m) ;(1, j, l)]_{q} } & =[(i, m) ;(j, l)]_{q} X_{11}-q^{-1}[(1, m) ;(j, l)]_{q} X_{i 1}+q^{-2}[(1, i) ;(j, l)]_{q} X_{m 1} \\
& =q^{-2} X_{m 1}[(1, i) ;(j, l)]_{q}-q^{-1} X_{m j}[(1, i) ;(1, l)]_{q}+X_{m l}[(1, i) ;(1, j)]_{q} .
\end{aligned}
$$

D'après le premier cas, $[(1, m) ;(j, l)]_{q} \in \tilde{I}_{q}^{\mathrm{g}}(\mathbf{X})$. Il s'ensuit $[(i, m) ;(j, l)]_{q} \in \tilde{I}_{q}^{\sharp}(\mathbf{X})$.

Remarque 2.2.4. (1) La preuve de la proposition 2.2 .3 montre en fait que, pour $2 \leq s \leq n: \tilde{I}_{q}\left(\mathbf{X}^{\prime}{ }_{(1, \ldots, s)}\right)=\left(\min _{q}\left(\mathbf{X}^{\prime}\right),[(1, m) ;(1,2)]_{q}, \ldots,[(1, m) ;(1, s)]_{q}\right)$.

(2) En itérant 2.2.3, on montre que $\tilde{I}_{q}(\mathbf{X})=\left([(1, k) ;(1, l)]_{q}, 2 \leq k \leq m, 2 \leq l \leq n\right)$.

Proposition 2.2.5. Dans les notations précédentes et pour $1 \leq s \leq n-1$ :

(1) $\tilde{I}_{q}\left(\mathbf{X}^{\prime}\right) \triangleleft \mathbb{K}_{q}\left[\mathbf{X}^{\prime}\right]_{X_{11}}$ est $\left(\sigma_{m 1}, \delta_{m 1}\right)$-stable, $\tilde{I}_{q}\left(\mathbf{X}_{(1, \ldots, s)}^{\prime}\right) \triangleleft \mathbb{K}_{q}\left[\mathbf{X}_{[1, \ldots, s]}^{\prime}\right]_{X_{11}}$ est $\left(\sigma_{m, s+1}, \delta_{m, s+1}\right)-$ stable;

(2) dans $\mathbb{K}_{q}\left[\mathbf{X}_{(1, \ldots, s+1)}^{\prime}\right]_{X_{11}},[(1, m) ;(1, s+1)]_{q}$ est normal modulo l'idéal $\left(\min _{q}\left(\mathbf{X}_{(1, \ldots, s)}^{\prime}\right)\right.$.

Preuve. La preuve repose sur le procédé décrit en 2.1.8 et les relations (4) à (8).

Soient $1 \leq s \leq n-1$ et $M=[(i, k) ;(j, l)]_{q} \in \min _{q}\left(\mathbf{X}_{(1, \ldots, s)}^{\prime}\right)$. Les relations (4) à (7) prouvent qu'il existe $\lambda \in \mathbb{K}^{*}$ tel que $X_{m, s+1} M-\lambda M X_{m, s+1} \in \tilde{I}_{q}\left(\mathbf{X}_{(1, \ldots, s)}^{\prime}\right) \triangleleft \mathbb{K}_{q}\left[\mathbf{X}_{(1, \ldots, s)}^{\prime}\right]_{X_{11}}$. Ceci montre que l'idéal $\tilde{I}_{q}\left(\mathbf{X}_{(1, \ldots, s)}^{\prime}\right)$ de $\mathbb{K}_{q}\left[\mathbf{X}_{(1, \ldots, s)}\right]_{X_{11}}$ est $\left(\sigma_{m, s+1}, \delta_{m, s+1}\right)$-stable. Les mêmes relations montrent que l'idéal $\tilde{I}_{q}\left(\mathbf{X}^{\prime}\right) \triangleleft \mathbb{K}_{q}\left[\mathbf{X}^{\prime}\right]_{X_{11}}$ est $\left(\sigma_{m 1}, \delta_{m 1}\right)$-stable. On a donc prouvé le (1).

Soient à présent $1 \leq s \leq n-1, M^{\prime}=[(1, m) ;(1, s+1)]_{q}$ et $X_{i j}$ un générateur canonique de $\mathbb{K}_{q}\left[X_{(1, \ldots, s+1]}^{\prime}\right]_{X_{11}}$. Nous allons montrer que $M^{\prime}$ et $X_{i j}$ commutent à un scalaire près modulo $\left(\min _{q}\left(\mathbf{X}^{\prime}(1, \ldots, s)\right)\right.$ dans $\mathbb{K}_{q}\left[\mathbf{X}_{[1, \ldots, s+1]}^{\prime}\right]_{X_{11}}$, prouvant ainsi (2).

Si $j>s+1$ et $1<i<m$, d'après les relations entre $A(23)$ et $X_{23}, M^{\prime} X_{i j}-q^{2} X_{i j} M^{\prime} \in$ $\left(\min _{q}\left(\mathbf{X}^{\prime}\right)\right) \triangleleft \mathbb{K}_{q}\left[\mathbf{X}^{\prime}(1, \ldots, s+1)\right]_{X_{11}}$.

Si $1<j<s+1$ et $1<i<m$, d'après les relations entre $A(22)$ et $X_{22}, M^{\prime} X_{i j}-q^{2} X_{i j} M^{\prime} \in$ $\left(\min _{q}\left(\mathbf{X}_{(1, \ldots, s)}^{\prime}\right)\right) \triangleleft \mathbb{K}_{q}\left[\mathbf{X}_{(1, \ldots, \ldots+1)}^{\prime}\right]_{X_{11}}$.

$\mathrm{Si} i=1$ (resp. $i=m$ ) et $1<j<s+1$, d'après les relations entre $A(22)$ et $X_{12}$ (resp. $X_{32}$ ), $M^{\prime} X_{i j}-q X_{i j} M^{\prime} \in\left([(1, m) ;(1,2)]_{q}, \ldots,[(1, m) ;(1, s)]_{q}\right) \triangleleft \mathbb{K}_{q}\left[\mathbf{X}_{(1, \ldots, s+1)}^{\prime}\right]_{X_{11}}$.

Si $j=1$ (resp. $j=s+1$ ) et $1<i<m$, d'après les relations entre $A(22)$ et $X_{21}$ (resp. $\left.X_{23}\right), M^{\prime} X_{i j}-q X_{i j} M^{\prime} \in\left(\min _{q}\left(\mathbf{X}^{\prime}\right)\right) \triangleleft \mathbb{K}_{q}\left[\mathbf{X}_{(1, \ldots,+1)}^{\prime}\right]_{X_{11}}$.

Si $i=1$ et $j>s+1$, d'après les relations entre $A(23)$ et $X_{13}, M^{\prime} X_{i j}-q X_{i j} M^{\prime}=0$.

Si $(i, j)=(1,1),(1, s+1),(m, 1)$ ou $(m, s+1)$, alors $M^{\prime} X_{i j}-X_{i j} M^{\prime}=0$.

Ceci achève la preuve.

Le lemme suivant est utile à la preuve de 2.2.7. Il est connu, nous n'en donnons pas la preuve.

Lemme 2.2.6. Soient $A$ un anneau, $\sigma$ un endomorphisme injectif de $A$ et $(\sigma, \delta)$ une dérivation tordue à gauche de $A$. On pose $B=A[X ; \sigma, \delta]$. 
(1) Si I est un idéal $(\sigma, \delta)$-stable de A, alors IB est un idéal bilatère de B et, si $(\bar{\sigma}, \bar{\delta})$ désigne la dérivation tordue induite par $(\sigma, \delta)$ sur $A / I$, l'application $\phi: B / I B \rightarrow(A / I)[X, \bar{\sigma}, \bar{\delta}]$ définie par $\phi\left(\left(\sum_{i=0}^{d} a_{i} X^{i}\right)+I B\right)=\sum_{i=0}^{d}\left(a_{i}+I\right) X^{i}$ est un isomorphisme d'anneaux.

(2) Si a est une unité de $A$ et si $b \in A$ est tel que $a X+b$ soit normal dans $B$, alors le morphisme canonique $A \hookrightarrow B \rightarrow B /(a X+b)$ est un isomorphisme.

Théorème 2.2.7. L'algèbre $\mathbb{K}_{q}[\mathbf{X}]_{X_{11}} / \tilde{I}_{q}(\mathbf{X})$ est intègre et un ordre maximal de son corps de fractions.

Preuve. Dans cette preuve, nous utiliserons 2.2.6 sans le mentionner explicitement. Nous notons $\tilde{J}_{q}\left(\mathbf{X}^{\prime}{ }_{11, \ldots, s+1\}}\right)$ l'idéal de $\mathbb{K}_{q}\left[\mathbf{X}^{\prime}{ }_{11, \ldots, s+1\}}\right]_{X_{11}}$ défini, pour $1 \leq s \leq n-1$, par:

$$
\begin{aligned}
& \tilde{J}_{q}\left(\mathbf{X}_{\{1, \ldots, s+1\}}^{\prime}\right)=\left(\min _{q}\left(\mathbf{X}^{\prime}\right),[(1, m) ;(1, l)]_{q}, 2 \leq l \leq s\right) \triangleleft \mathbb{K}_{q}\left[\mathbf{X}_{\{1, \ldots, s+1\}}^{\prime}\right]_{X_{11}}, \text { si } s \neq 1, \\
& \tilde{J}_{q}\left(\mathbf{X}_{\{1,2\}}^{\prime}\right)=\left(\min _{q}\left(\mathbf{X}^{\prime}\right)\right) \triangleleft \mathbb{K}_{q}\left[\mathbf{X}_{\{1,2\}}^{\prime}\right]_{X_{11}} .
\end{aligned}
$$

D'après 2.2.5, $\tilde{I}_{q}\left(\mathbf{X}^{\prime}\right)$ est $\left(\sigma_{m 1}, \delta_{m 1}\right)$-stable. Ainsi, $\tilde{I}_{q}\left(\mathbf{X}_{\{1\}}^{\prime}\right)=\tilde{I}_{q}\left(\mathbf{X}^{\prime}\right) \mathbb{K}_{q}\left[\mathbf{X}_{\{1\}}^{\prime}\right]_{X_{11}}$, et (avec des notations évidentes) il existe un isomorphisme

$$
\mathbb{K}_{q}\left[\mathbf{X}_{\{11}^{\prime}\right]_{X_{11}} / \tilde{I}_{q}\left(\mathbf{X}_{\langle 1\}}^{\prime}\right) \stackrel{\phi_{1}}{\longrightarrow}\left(\mathbb{K}_{q}\left[\mathbf{X}^{\prime}\right]_{X_{11}} / \tilde{I}_{q}\left(\mathbf{X}^{\prime}\right)\right)\left[X_{m 1} ; \bar{\sigma}_{m 1}, \bar{\delta}_{m 1}\right]
$$

tel que $\phi_{1}\left(X_{i j}+\tilde{I}_{q}\left(\mathbf{X}_{\{1\}}^{\prime}\right)\right)=X_{i j}+\tilde{I}_{q}\left(\mathbf{X}^{\prime}\right)$ pour $i<m$ et $\phi_{1}\left(X_{m 1}+\tilde{I}_{q}\left(\mathbf{X}^{\prime}{ }_{(1)}\right)\right)=X_{m 1}$.

D'après 2.2.5, si $s \in \mathbb{N}_{n-1}, \tilde{I}_{q}\left(\mathbf{X}_{11, \ldots, s)}^{\prime}\right)$ est un idéal $\left(\sigma_{m, s+1}, \delta_{m, s+1}\right)$-stable de $\mathbb{K}_{q}\left[\mathbf{X}_{(1, \ldots, s)}^{\prime}\right]_{X_{11}}$. Par suite, d'après 2.2.4, $\tilde{J}_{q}\left(\mathbf{X}_{\{1, \ldots, s+1\}}^{\prime}\right)=\tilde{I}_{q}\left(\mathbf{X}_{\{1, \ldots, s\}}^{\prime}\right) \mathbb{K}_{q}\left[\mathbf{X}_{\{1, \ldots, s+1\}}^{\prime}\right]_{X_{11}}$. De plus, 2.2 .5 assure alors que $[(1, m) ;(1, s+1)]_{q}$ est normal modulo $\tilde{J}_{q}\left(\mathbf{X}_{\{1, \ldots, s+1\}}^{\prime}\right)$ dans $\mathbb{K}_{q}\left[\mathbf{X}_{(1, \ldots, s+1)}^{\prime}\right]_{x_{11}}$. Donc, d'après 2.2.4, $\tilde{I}_{q}\left(\mathbf{X}_{\{1, \ldots, s+1\}}^{\prime}\right)=\tilde{J}_{q}\left(\mathbf{X}_{\{1, \ldots, s+1\}}^{\prime}\right)+[(1, m) ;(1, s+1)]_{q} \mathbb{K}_{q}\left[\mathbf{X}_{(1, \ldots, s+1\}}^{\prime}\right]_{X_{11}}$. D'autre part, il existe un isomorphisme

$$
\mathbb{K}_{q}\left[\mathbf{X}_{(1, \ldots, s+1)}^{\prime}\right]_{X_{11}} / \tilde{J}_{q}\left(\mathbf{X}_{\{1, \ldots, s+1)}^{\prime}\right) \stackrel{\phi_{s+1}}{\longrightarrow} \mathbb{K}_{q}\left[\mathbf{X}_{(1, \ldots, s\}}^{\prime}\right]_{X_{11}} / \tilde{I}_{q}\left(\mathbf{X}_{(1, \ldots, s)}^{\prime}\right)\left[X_{m, s+1} ; \bar{\sigma}_{m, s+1}, \bar{\delta}_{m, s+1}\right]
$$

tel que $\phi_{s+1}\left(X_{i j}+\tilde{J}_{q}\left(\mathbf{X}_{(1, \ldots, s+1)}^{\prime}\right)\right)=X_{i j}+\tilde{I}_{q}\left(\mathbf{X}_{(1, \ldots, s)}^{\prime}\right)$ pour $i<m$ ou $j<s+1$ et $\phi_{s+1}\left(X_{m, s+1}+\right.$ $\tilde{J}_{q}\left(\mathbf{X}_{\{1, \ldots, s+1)}^{\prime}\right)=X_{m, s+1}$.

Comme $[(1, m) ;(1, s+1)]_{q}=X_{11} X_{m, s+1}-q X_{m 1} X_{1, s+1}$ est normal modulo $\tilde{J}_{q}\left(\mathbf{X}_{(1, \ldots, s+1)}^{\prime}\right)$, $\phi_{s+1}\left(X_{11}+\tilde{J}_{q}\left(\mathbf{X}_{\{1, \ldots, s+1)}^{\prime}\right)\right) X_{m, s+1}-q \phi_{s+1}\left(X_{m 1}+\tilde{J}_{q}\left(\mathbf{X}^{\prime}{ }_{11, \ldots, s+1)}\right)\right) \phi_{s+1}\left(X_{1, s+1}+\tilde{J}_{q}\left(\mathbf{X}_{\{1, \ldots, s+1)}^{\prime}\right)\right)$ est normal dans $\mathbb{K}_{q}\left[\mathbf{X}_{(1, \ldots, s)}^{\prime}\right]_{X_{11}} / \tilde{I}_{q}\left(\mathbf{X}_{(1, \ldots, s)}^{\prime}\right)\left[X_{m, s+1} ; \bar{\sigma}_{m, s+1}, \bar{\delta}_{m, s+1}\right]$. Comme enfin $\phi_{s+1}\left(X_{11}+\tilde{J}_{q}\left(\mathbf{X}_{(1, \ldots, s+1)}^{\prime}\right)\right)=$ $X_{11}+\tilde{I}_{q}\left(\mathbf{X}_{(1, \ldots, s)}^{\prime}\right)$ est une unité de $\mathbb{K}_{q}\left[\mathbf{X}_{(1, \ldots, s)}^{\prime}\right]_{X_{11}} / \tilde{I}_{q}\left(\mathbf{X}_{(1, \ldots, s)}^{\prime}\right)$, on a donc:

$$
\mathbb{K}_{q}\left[\mathbf{X}_{\{1, \ldots, s+1\}}^{\prime}\right]_{X_{11}} / \tilde{I}_{q}\left(\mathbf{X}_{\{1, \ldots, s+1\}}^{\prime}\right) \cong \mathbb{K}_{q}\left[\mathbf{X}_{\{1, \ldots, s\}}^{\prime}\right]_{X_{11}} / \tilde{I}_{q}\left(\mathbf{X}_{\{1, \ldots, s)}^{\prime}\right)
$$

Les isomorphismes établis ci-dessus conduisent alors à

$$
\mathbb{K}_{q}\left[\mathbf{X}_{\{1, \ldots, n\}}^{\prime}\right]_{X_{11}} / \bar{I}_{q}\left(\mathbf{X}_{\{1, \ldots, n\}}^{\prime}\right) \cong \mathbb{K}_{q}\left[\mathbf{X}^{\prime}{ }_{[1\}}\right]_{X_{11}} / \tilde{I}_{q}\left(\mathbf{X}^{\prime}{ }_{(1)}\right) \cong\left(\mathbb{K}_{q}\left[\mathbf{X}^{\prime}\right]_{X_{11}} / \tilde{I}_{q}\left(\mathbf{X}^{\prime}\right)\right)\left[X_{m 1} ; \bar{\sigma}_{m 1}, \bar{\delta}_{m 1}\right]
$$


On peut maintenant montrer le résultat annoncé. On fixe un entier non nul $n$ et on procède par récurrence sur $m$. L'assertion est vraie lorsque $m=1$ puisque l'idéal de $\mathcal{O}_{q}(M(1, n))_{X_{11}}$ engendré par les mineurs quantiques $2 \times 2$ est réduit à (0). Supposons le résultat acquis jusqu'à l'ordre $m-1$. D'après ce qui précède, on a

$$
\mathbb{K}_{q}[\mathbf{X}]_{X_{11}} / \tilde{I}_{q}(\mathbf{X})=\mathbb{K}_{q}\left[\mathbf{X}^{\prime}{ }_{11, \ldots, n]}\right]_{X_{11}} / \tilde{I}_{q}\left(\mathbf{X}_{\{1, \ldots, n\}}^{\prime}\right) \cong\left(\mathbb{K}_{q}\left[\mathbf{X}^{\prime}\right]_{X_{11}} / \tilde{I}_{q}\left(\mathbf{X}^{\prime}\right)\right)\left[X_{m 1} ; \bar{\sigma}_{m 1}, \bar{\delta}_{m 1}\right]
$$

L'hypothèse de récurrence assure que $\mathbb{K}_{q}\left[\mathbf{X}^{\prime}\right]_{X_{11}} / \tilde{I}_{q}\left(\mathbf{X}^{\prime}\right)$ est un anneau intègre, ordre maximal de son corps de fractions. Comme $\bar{\sigma}_{m 1}$ est un automorphisme, $\mathbb{K}_{q}[\mathbf{X}]_{X_{11}} / \tilde{I}_{q}(\mathbf{X})$ est intègre et, d'après 1.3 , c'est un ordre maximal de son corps de fractions. Ceci achève la preuve.

Remarque 2.2.8. A l'aide d'une récurrence, on déduit de la preuve de 2.2.7 que $\mathbb{K}_{q}[\mathbf{X}]_{X_{11}} / \tilde{I}_{q}(\mathbf{X})$ est isomorphe à la sous-algèbre de $\mathbb{K}_{q}[\mathbf{X}]_{X_{11}}$ engendrée par $X_{11}^{ \pm 1}$, les $X_{1 j}$ et les $X_{i 1}$, pour $2 \leq i \leq m$ et $2 \leq j \leq n$. Ainsi, $\mathbb{K}_{q}[\mathbf{X}]_{X_{11}} / \tilde{I}_{q}(\mathbf{X})$ est isomorphe à un localisé d'espace quantique.

\subsection{Intégrité et normalité de $R_{q}(X)$}

Dans le premier paragraphe de cette sous-section, on montre que l'anneau $R_{q}(\mathbf{X})$ (défini en 2.1.1) est intègre (Théo. 2.3.8). C'est trivial lorsque $\mathbf{X}$ est une matrice à une ligne et une colonne. La démonstration de l'intégrité de $R_{q}(\mathbf{X})$ procède par récurrence sur la somme du nombre de lignes et du nombre de colonnes de X. Avec les notations précédemment introduites, il suffit donc de montrer que si l'on fait l'hypothèse (H) suivante:

Hypothèse: Les anneaux $R_{q}\left(\mathbf{X}^{\prime}\right)$ et $R_{q}\left(\mathbf{X}^{\prime \prime}\right)$ sont intègres,

alors $R_{q}(\mathbf{X})$ est un anneau intègre.

Dans le second paragraphe, on montre que $R_{q}(\mathbf{X})$ est un ordre maximal de son corps de fractions (Théo. 2.3.11).

Intégrité de $R_{q}(\mathrm{X})$. Dans ce paragraphe, on suppose l'hypothèse $(\mathrm{H})$ satisfaite. On reprend les notations de 2.1 et on considère les idéaux suivants de $\mathbb{K}_{q}[X]$ :

$$
L_{q}(\mathbf{X})=\left(\min _{q}\left(\mathbf{X}^{\prime}\right), X_{m 1}, X_{m 2}, \ldots, X_{m n}\right) \text { et } C_{q}(\mathbf{X})=\left(\min _{q}\left(\mathbf{X}^{\prime \prime}\right), X_{1 n}, X_{2 n}, \ldots, X_{m n}\right) \text {. }
$$

Proposition 2.3.1. On a:

(1) $L_{q}(\mathbf{X})=I_{q}\left(\mathbf{X}^{\prime}\right) \mathbb{K}_{q}[\mathbf{X}]+\mathbb{K}_{q}[\mathbf{X}] X_{m 1}+\mathbb{K}_{q}[\mathbf{X}] X_{m 2}+\ldots+\mathbb{K}_{q}[\mathbf{X}] X_{m n}$,

(2) $C_{q}(\mathbf{X})=I_{q}\left(\mathbf{X}^{\prime \prime}\right) \mathbb{K}_{q}[\mathbf{X}]+\mathbb{K}_{q}[\mathbf{X}] X_{1 n}+\mathbb{K}_{q}[\mathbf{X}] X_{2 n}+\ldots+\mathbb{K}_{q}[\mathbf{X}] X_{m n}$.

Preuve. En procédant comme en 2.2.5, on montre aisément que $I_{q}\left(\mathbf{X}^{\prime}\right)$ est $\left(\sigma_{m 1}, \delta_{m)}\right)$ stable et que pour $1 \leq s \leq n-1$, l'idéal $\left(\min _{q}\left(\mathbf{X}^{\prime}\right)\right) \triangleleft \mathbb{K}_{q}\left[\mathbf{X}_{[1, \ldots, s\}}^{\prime}\right]$ est stable sous 
$\left(\sigma_{m, s+1}, \delta_{m, s+1}\right)$. Il s'ensuit que $\left(\min _{q}\left(\mathbf{X}^{\prime}\right)\right)=I_{q}\left(\mathbf{X}^{\prime}\right) \mathbb{K}_{q}[\mathbf{X}] \triangleleft \mathbb{K}_{q}[\mathbf{X}]$. D'autre part, il est clair par définition de $\mathbb{K}_{q}[\mathbf{X}]$ que le suite $\left\{X_{m 1}, X_{m 2}, \ldots, X_{m n}\right\}$ est une suite normalisante de $\mathbb{K}_{q}[\mathbf{X}]$. Le point (1) s'ensuit immédiatement. A l'aide de l'isomorphisme $\operatorname{Tr} \mathrm{de}$ transposition, on en déduit (2).

Proposition 2.3.2. Les idéaux $L_{q}(\mathbf{X})$ et $C_{q}(\mathbf{X})$ de $\mathbb{K}_{q}[\mathbf{X}]$ sont complètement premiers.

Preuve. Il est clair que $\mathbb{K}_{q}[\mathbf{X}] / L_{q}(\mathbf{X}) \cong \mathbb{K}_{q}\left[\mathbf{X}^{\prime}\right] / I_{q}\left(\mathbf{X}^{\prime}\right)$ et $\mathbb{K}_{q}[\mathbf{X}] / C_{q}(\mathbf{X}) \cong \mathbb{K}_{q}\left[\mathbf{X}^{\prime \prime}\right] / I_{q}\left(\mathbf{X}^{\prime \prime}\right)$. L'hypothèse $(\mathbf{H})$ assure donc que $L_{q}(\mathbf{X})$ et $C_{q}(\mathbf{X})$ sont complètement premiers.

Lemme 2.3.3. Soit $G \in C_{q}(\mathbf{X})$; alors, il existe $H \in I_{q}\left(\mathbf{X}^{\prime \prime}\right) \triangleleft \mathbb{K}_{q}\left[\mathbf{X}^{\prime \prime}\right], H_{1}, \ldots, H_{m-1} \in \mathbb{K}_{q}[\mathbf{X}]$ et $H_{m} \in \oplus_{i=0}^{\infty} \mathbb{K}_{q}\left[\mathbf{X}^{\prime \prime}\right] X_{m n}^{i}$ tels que $G=H+H_{1} X_{1 n}+H_{2} X_{2 n}+\ldots+H_{m} X_{m n}$.

Preuve. D'après 2.3.1, $C_{q}(\mathbf{X})=I_{q}\left(\mathbf{X}^{\prime \prime}\right) \mathbb{K}_{q}[\mathbf{X}]+\mathbb{K}_{q}[\mathbf{X}] X_{1 n}+\ldots+\mathbb{K}_{q}[\mathbf{X}] X_{m n}$. D'autre part, il est clair d'après (2) que $I_{q}\left(\mathbf{X}^{\prime \prime}\right) \mathbb{K}_{q}[\mathbf{X}] \subseteq I_{q}\left(\mathbf{X}^{\prime \prime}\right)+\mathbb{K}_{q}[\mathbf{X}] X_{1 n}+\ldots+\mathbb{K}_{q}[\mathbf{X}] X_{m n}$. Par suite, on a $C_{q}(\mathbf{X})=I_{q}\left(\mathbf{X}^{\prime \prime}\right)+\mathbb{K}_{q}[\mathbf{X}] X_{1 n}+\ldots+\mathbb{K}_{q}[\mathbf{X}] X_{m n}$.

Ainsi, si $G \in C_{q}(\mathbf{X}), \quad G=H+H_{1} X_{1 n}+\ldots+H_{m} X_{m n}$, où $H \in I_{q}\left(\mathbf{X}^{\prime \prime}\right)$ et $H_{i} \in \mathbb{K}_{q}[\mathbf{X}]$, $1 \leq i \leq m$. Compte tenu de (2) et du fait que, pour $1 \leq i \leq m$, les $X_{i n}$ commutent deuxà-deux à un scalaire près, on peut supposer que $H_{m} \in \oplus_{i=0}^{\infty} \mathbb{K}_{q}\left[\mathbf{X}^{\prime \prime}\right] X_{m n}^{i}$, ce qui achève la preuve.

Nous montrons, à présent, que l'idéal $\left(\min _{q}(\mathbf{X}), X_{m n}\right)$ de $\mathbb{K}_{q}[\mathbf{X}]$ est semi-premier (Théo. 2.3.5).

Lemme 2.3.4. Les idéaux premiers minimaux au-dessus de $\left(\min _{q}(\mathbf{X}), X_{m n}\right)$ sont les idéaux complètement premiers $L_{q}(\mathbf{X})$ et $C_{q}(\mathbf{X})$.

Preuve. Il est clair que $\left(\min _{q}(\mathbf{X}), X_{m n}\right) \subseteq L_{q}(\mathbf{X}) \cap C_{q}(\mathbf{X})$. Soit $P$ un idéal premier minimal au dessus de $\left(\min _{q}(\mathbf{X}), X_{m n}\right)$. Si $i \in \mathbb{N}_{m-1}$ et $j \in \mathbb{N}_{n-1}$, comme $X_{m n}$ et le mineur quantique $[(i, m) ;(j, n)]_{q}=X_{i j} X_{m n}-q X_{m j} X_{\text {in }}$ sont dans $P$, on a $X_{m j} X_{i n} \in P$.

1-er cas: $X_{1 n} \notin P$. Alors, pour $j \in \mathbb{N}_{n-1}, X_{m j} X_{1 n} \in P$ et comme $X_{1 n}$ est un élément normal de $\mathbb{K}_{q}[\mathbf{X}]$, on a $X_{m j} \in P$. Ainsi, $P$ contient $L_{q}(\mathbf{X})$. D'après 2.3.2, la minimalité de $P$ conduit à $P=L_{q}(\mathbf{X})$.

2-ème cas: $X_{1 n} \in P$. Nous allons montrer qu'alors $X_{\text {in }} \in P$ pour $i \in \mathbb{N}_{m-1}$. Remarquons d'abord que l'isomorphisme $\mathbb{K}_{q}[\mathbf{X}] / L_{q}(\mathbf{X}) \cong \mathbb{K}_{q}\left[\mathbf{X}^{\prime}\right] / I_{q}\left(\mathbf{X}^{\prime}\right)$ joint à 2.1 .2 assure que $X_{1 n} \notin L_{q}(\mathbf{X})$. A présent, supposons que $X_{2 n} \notin P$. Alors, comme $X_{2 n}$ est normal modulo $X_{1 n}$, les relations $X_{m j} X_{2 n} \in P, j \in \mathbb{N}_{n-1}$, conduisent à $X_{m j} \in P$, pour $j \in \mathbb{N}_{n-1}$. Mais alors $L_{q}(\mathbf{X}) \subseteq P$ et, par minimalité de $P, L_{q}(\mathbf{X})=P$. Ceci conduit à $X_{1 n} \in L_{q}(\mathbf{X})$ qui est une contradiction. Ainsi, $X_{2 n} \in P$. Comme $\left\{X_{1 n}, X_{2 n}, \ldots, X_{m-1, n}\right\}$ est une suite normalisante, on peut itérer ce procédé et conclure que $X_{2 n}, \ldots, X_{m-1, n} \in P$. Finalement $X_{1 n}, \ldots, X_{m n} \in P$ et donc $C_{q}(\mathbf{X}) \subseteq P$. Puisque $P$ est minimal, d'après 2.3.2, il vient $C_{q}(\mathbf{X})=P$. Ceci achève la preuve.

Théorème 2.3.5. Dans $\mathbb{K}_{q}[\mathrm{X}]$, on $a\left(\min _{q}(\mathbf{X}), X_{m n}\right)=L_{q}(\mathbf{X}) \cap C_{q}(\mathbf{X})$. 
Preuve. Compte tenu de 2.3.4, il reste à montrer que $\left(\min _{q}(\mathbf{X}), X_{m n}\right) \supseteq L_{q}(\mathbf{X}) \cap C_{q}(\mathbf{X})$. Soit $F$ dans $L_{q}(\mathbf{X}) \cap C_{q}(\mathbf{X})$; d'après 2.3.1, $F=F^{\prime}+F^{\prime \prime}$, où $F^{\prime} \in I_{q}\left(\mathbf{X}^{\prime}\right) \mathbb{K}_{q}[\mathbf{X}] \subseteq\left(\min _{q}(\mathbf{X})\right.$, $\left.X_{m n}\right)$ et $F^{\prime \prime}=\sum_{j=1}^{n} F_{j} X_{m j}$, avec $F_{j} \in \mathbb{K}_{q}[\mathbf{X}]$. Il suffit de montrer que $F^{\prime \prime} \in\left(\min _{q}(\mathbf{X}), X_{m n}\right)$. De plus, (2) assure que, pour $j \in \mathbb{N}_{n}, F_{j}=G_{j}+\sum_{i=1}^{m} G_{i j} X_{\text {in }}$, où $G_{j} \in \mathbb{K}_{q}\left[\mathbf{X}^{\prime \prime}\right]$ et $G_{i j} \in \mathbb{K}_{q}[\mathbf{X}]$. Donc, $F^{\prime \prime}=G+G^{\prime}$, où $G=\sum_{j=1}^{n} G_{j} X_{m j}$ et $G^{\prime}=\sum_{j=1}^{n} \sum_{i=1}^{m} G_{i j} X_{i n} X_{m j}$. Il vient,

$$
\begin{aligned}
G^{\prime}= & \sum_{j=1}^{n} \sum_{i=1}^{m} G_{i j} X_{i n} X_{m j} \\
= & \sum_{j=1}^{n-1} \sum_{i=1}^{m-1} G_{i j} X_{i n} X_{m j}+\sum_{i=1}^{m-1} G_{i n} X_{i n} X_{m n}+\sum_{j=1}^{n-1} G_{m j} X_{m n} X_{m j}+G_{m n} X_{m n} X_{m n} \\
= & \sum_{j=1}^{n-1} \sum_{i=1}^{m-1} G_{i j} q^{-1}\left(X_{i j} X_{m n}-[(i, m) ;(j, n)]_{q}\right) \\
& +\sum_{i=1}^{m-1} G_{i n} X_{i n} X_{m n}+\sum_{j=1}^{n-1} G_{m j} X_{m n} X_{m j}+G_{m n} X_{m n} X_{m n} .
\end{aligned}
$$

Ainsi, $G^{\prime} \in\left(\min _{q}(\mathbf{X}), X_{m n}\right) \subseteq L_{q}(\mathbf{X}) \cap C_{q}(\mathbf{X})$ et par suite $G=F^{\prime \prime}-G^{\prime}=F-F^{\prime}-G^{\prime} \in$ $L_{q}(\mathbf{X}) \cap C_{q}(\mathbf{X})$. En particulier $G \in C_{q}(\mathbf{X})$ et d'après 2.3.3, il existe $H \in I_{q}\left(\mathbf{X}^{\prime \prime}\right) \triangleleft \mathbb{K}_{q}\left[\mathbf{X}^{\prime \prime}\right]$, $H_{m} \in \oplus_{i=0}^{\infty} \mathbb{K}_{q}\left[\mathbf{X}^{\prime \prime}\right] X_{m n}^{i}$ et $H_{i} \in \mathbb{K}_{q}[\mathbf{X}]$, pour $i \in \mathbb{N}_{m-1}$, tels que $G=H+\sum_{i=1}^{m} H_{i} X_{i n}$. D'autre part, $G=\sum_{j=1}^{n} G_{j} X_{m j} \in \mathbb{K}_{q}\left[\mathbf{X}^{\prime \prime}\right] \oplus \mathbb{K}_{q}\left[\mathbf{X}^{\prime \prime}\right] X_{m n}$, donc

$$
G-H-H_{m} X_{m n}=\sum_{i=1}^{m-1} H_{i} X_{i n} \in \oplus_{i=0}^{\infty} \mathbb{K}_{q}\left[\mathbf{X}^{\prime \prime}\right] X_{m n}^{i} .
$$

Enfin, $\mathbb{K}_{q}[\mathbf{X}]$ est un $\mathbb{K}_{q}\left[\mathbf{X}^{\prime \prime}\right]$-module libre à gauche de base $\left\{X_{1 n}^{i_{1}} \ldots X_{m n}^{i_{n}},\left(i_{1}, \ldots, i_{m}\right) \in \mathbb{N}^{m}\right\}$ (voir (2)). Compte tenu des relations de commutation à un scalaire non nul près entre les $X_{\text {in }}$, il est clair que $\sum_{i=1}^{m-1} H_{i} X_{i n}$ peut s'écrire comme combinaison linéaire à coefficients dans $\mathbb{K}_{q}\left[\mathbf{X}^{\prime \prime}\right]$ d'éléments $X_{1 n}^{i,} \ldots X_{m n}^{i_{n}}$ où $i_{1}+\ldots+i_{m-1} \neq 0$. Puisque $G-H-H_{m} X_{m n} \in \oplus_{i=0}^{\infty} \mathbb{K}_{q}\left[\mathbf{X}^{\prime \prime}\right] X_{m n}^{i}$, il vient $G-H-H_{m} X_{m n}=0$ et donc $G \in\left(\min _{q}(\mathbf{X}), X_{m n}\right)$. Ainsi, $F^{\prime \prime} \in\left(\min _{q}(\mathbf{X}), X_{m n}\right)$.

On peut, à présent, montrer que $R_{q}(\mathrm{X})$ est intègre.

Lemme 2.3.6. Soit $F \in \mathbb{K}_{q}[\mathbf{X}]$. Si il existe $p \in \mathbb{N}$ tel que $X_{m n}^{p} F \in I_{q}(\mathbf{X})$, alors il existe $r \in \mathbb{N}$ tel que $F X_{11}^{r} \in I_{q}(\mathbf{X})$.

Preuve. On a une injection canonique $\mathbb{K}_{q}[\mathbf{X}] \hookrightarrow \mathbb{K}_{q}[\mathbf{X}]_{X_{11}}$ et $I_{q}(\mathbf{X}) \mathbb{K}_{q}[\mathbf{X}]_{X_{11}}=\tilde{I}_{q}(\mathbf{X})$ (voir 2.2.2). Notons que $X_{m n} \notin \tilde{I}_{q}(\mathbf{X})$. En effet, dans le cas contraire, on aurait $X_{m 1} X_{1 n} \in \tilde{I}_{q}(\mathbf{X})$ et donc $X_{m 1} \in \tilde{I}_{q}(\mathbf{X})$ ou $X_{1 n} \in \tilde{I}_{q}(\mathbf{X})$ (cf. 2.2.7), contredisant 2.2.2.

Soit alors $F \in \mathbb{K}_{q}[\mathbf{X}]$; si il existe $p \in \mathbb{N}$ tel que $X_{m n}^{p} F \in I_{q}(\mathbf{X})$, on a $X_{m n}^{p} F \in \tilde{I}_{q}(\mathbf{X})$. Comme $X_{m n} \notin \tilde{I}_{q}(\mathbf{X}), 2.2 .7$ assure que $F \in \tilde{I}_{q}(\mathbf{X})=I_{q}(\mathbf{X}) \mathbb{K}_{q}[\mathbf{X}]_{X_{11}}$. Par suite, il existe $r \in \mathbb{N}$ tel que $F X_{11}^{r} \in I_{q}(\mathbf{X})$.

Proposition 2.3.7. Soit $F \in \mathbb{K}_{q}[\mathbf{X}]$; si il existe $p \in \mathbb{N}$ tel que $F X_{11}^{p} \in I_{q}(\mathbf{X})$, alors $F \in I_{q}(\mathbf{X})$. En d'autres termes, $X_{11}$ est régulier à gauche modulo $I_{q}(\mathbf{X})$.

Preuve. On raisonne par récurrence sur le degré total de $F$ (noté $\operatorname{deg} F$ ). Soient 
$F \in \mathbb{K}_{q}[\mathbf{X}]$ et $p \in \mathbb{N}$ tels que $F X_{11}^{p} \in I_{q}(\mathbf{X})$. Si $\operatorname{deg} F=0, F \in \mathbb{K}$. Si $F \neq 0$, il s'ensuit que $X_{11}^{p} \in I_{q}(\mathbf{X})$, ce qui est faux d'après 2.1.2. Ainsi, l'assertion est vraie pour $\operatorname{deg} F=0$.

Soit $d \in \mathbb{N}$, on suppose le résultat acquis pour tout élément de degré au plus $d$ et on considère un élément $F \in \mathbb{K}_{q}[\mathbf{X}]$, de degré $d+1$ et tel que $F X_{11}^{p} \in I_{q}(\mathbf{X})$ pour un certain entier $p \in \mathbb{N}$. Alors, $F X_{11}^{p} \in L_{q}(\mathbf{X}) \cap C_{q}(\mathbf{X})$ (cf. 2.3.4). Comme $L_{q}(\mathbf{X})$ et $C_{q}(\mathbf{X})$ sont complètement premiers (cf. 2.3.2), et ne contiennent pas $X_{11}$, on a $F \in\left(I_{q}(\mathbf{X}), X_{m n}\right)$ d'après 2.3.5. D'après 2.1.3, $X_{m n}$ est normal modulo $I_{q}(\mathbf{X}), F$ peut donc s'écrire sous la forme $F=G+X_{m n} f$, où $G \in I_{q}(\mathbf{X})$ et $f \in \mathbb{K}_{q}[\mathbf{X}]$. De plus, comme $I_{q}(\mathbf{X})$ est engendré par des éléments homogènes de l'anneau $\mathbb{K}_{q}[\mathbf{X}]$ (gradué par le degré total), c'est un idéal gradué qui contient donc les composantes homogènes de ses éléments. Il s'ensuit que l'on peut supposer que $\operatorname{deg} f \leq d$.

Comme $F X_{11}^{p} \in I_{q}(\mathbf{X})$, il vient $X_{m n} f X_{11}^{p} \in I_{q}(\mathbf{X})$. Le lemme 2.3.6 assure alors qu'il existe $r \in \mathbb{N}$ tel que $f X_{11}^{r} \in I_{q}(\mathbf{X})$. Mais, par hypothèse de récurrence, ceci conduit à $f \in I_{q}(\mathbf{X})$, et finalement $F \in I_{q}(\mathbf{X})$.

Théorème 2.3.8. L'anneau $R_{q}(\mathrm{X})$ est intègre.

Preuve. Soient $m$ et $n$ deux entiers non nuls $(m, n \geq 2)$ et $\mathbf{X}=\left(X_{i j}\right)_{1 \leq i \leq m, 1 \leq j \leq n}$. Si l'on suppose $R_{q}\left(\mathbf{X}^{\prime}\right)$ et $R_{q}\left(\mathbf{X}^{\prime \prime}\right)$ intègres, 2.2.7 et 2.3.7 assurent que $R_{q}(\mathbf{X})$ est intègre. On peut alors conclure par récurrence comme indiqué dans l'introduction de cette soussection.

Normalité de $R_{q}(\mathbf{X})$. On montre à présent que l'anneau intègre $R_{q}(\mathbf{X})=\mathbb{K}_{q}[\mathbf{X}] / I_{q}(\mathbf{X})$ est un ordre maximal de son corps de fractions. On notera $x_{i j}$ la classe de $X_{i j}$ modulo $I_{q}(\mathbf{X})$. D'après 2.1.3, les $x_{i j} \in R_{q}(\mathbf{X})$ commutent deux-à-deux à un scalaire non nul près. Ils sont donc normaux.

Lemme 2.3.9. La partie multiplicative $\left\{x_{11}^{i}, i \in \mathbb{N}\right\}$ est un ensemble de dénominateurs de $R_{q}(\mathbf{X})$. De plus $R_{q}(\mathbf{X})_{x_{11}} \cong \mathbb{K}_{q}[\mathbf{X}]_{X_{11}} / \tilde{I}_{q}(\mathbf{X})$.

Preuve. Comme $x_{11}$ est normal dans $R_{q}(\mathbf{X}),\left\{x_{11}^{i}, i \in \mathbb{N}\right\}$ est un ensemble de dénominateurs de $R_{q}(\mathbf{X})$. De plus, le morphisme canonique $\mathbb{K}_{q}[\mathbf{X}] \rightarrow R_{q}(\mathbf{X}) \hookrightarrow R_{q}(\mathbf{X})_{x_{11}}$ induit un morphisme $\mathbb{K}_{q}[\mathbf{X}]_{X_{11}} \rightarrow R_{q}(\mathbf{X})_{x_{11}}$, clairement surjectif et donc le noyau est $\tilde{I}_{q}(\mathbf{X})$.

Proposition 2.3.10. L'ensemble $\left\{x_{m n}^{i}, i \in \mathbb{N}\right\}$ est un ensemble de dénominateurs de $R_{q}(\mathbf{X})$ et l'algèbre intègre $R_{q}(\mathbf{X})_{x_{-}}$est un ordre maximal de son corps de fractions.

Preuve. On reprend les notations de 2.1.7. D'après 2.3.9, $\left\{y_{11}^{i}, i \in \mathbb{N}\right\}$ est un ensemble de dénominateurs de $R_{q^{-1}}(\mathbf{Y})$ et $R_{q^{-1}}(\mathbf{Y})_{y_{11}} \cong \mathbb{K}_{q^{-1}}[\mathbf{Y}]_{Y_{11}} / \tilde{I}_{q^{-1}}(\mathbf{Y})$. D'après 2.2.7, $\boldsymbol{R}_{q^{-1}}(\mathbf{Y})_{y_{11}}$ est donc un ordre maximal de son corps de fractions. De plus, il est clair d'après 2.1.7 que l'isomorphisme $\mathbf{S}: \mathbb{K}_{q}[\mathbf{X}] \rightarrow \mathbb{K}_{q^{-1}}[\mathbf{Y}]$ induit un isomorphisme $\tilde{\mathbf{S}}: R_{q}(\mathbf{X})_{x_{m}} \rightarrow R_{q^{-1}}(\mathbf{Y})_{y_{11}}$. Ceci achève la preuve. 
Théorème 2.3.11. L'anneau intègre $R_{q}(\mathbf{X})$ est un ordre maximal de son corps de fractions.

Preuve. D'après 2.3.5, $\left(\min _{q}(\mathbf{X}), X_{m n}\right)=L_{q}(\mathbf{X}) \cap C_{q}(\mathbf{X}) \triangleleft \mathbb{K}_{q}[\mathbf{X}]$. Il s'ensuit que les idéaux $l_{q}(\mathbf{X})=\left(x_{m 1}, \ldots, x_{m n}\right)$ et $c_{q}(\mathbf{X})=\left(x_{1 n}, \ldots, x_{m n}\right)$ de $R_{q}(\mathbf{X})$, qui sont complètement premiers d'après 2.3.2, satisfont à $\left(x_{m n}\right)=l_{q}(\mathbf{X}) \cap c_{q}(\mathbf{X})$. De plus, on a déjà vu que $x_{m n}$ est un élément normal de $R_{q}(\mathbf{X})$. Enfin, les relations de commutation entre $x_{m n}$ et les générateurs de $l_{q}(\mathbf{X})$ et $c_{q}(\mathbf{X})$ (qui se déduisent de la définition de $\mathbb{K}_{q}[\mathbf{X}]$ ) montrent que ces deux idéaux sont stables sous l'automorphisme associé à l'élément normal régulier $x_{m n}$ de $R_{q}(\mathbf{X})$. Comme d'après 2.3.10 $R_{q}(\mathbf{X})_{x_{m m}}$ est un ordre maximal de son corps de fractions, on est dans les hypothèses du lemme 1.1 qui permet de conclure que $R_{q}(\mathbf{X})$ est un ordre maximal de son corps de fractions.

\section{Espaces quantiques uniparamétrés}

Dans cette section, nous étudions le cas de l'espace quantique uniparamétré $E_{n}^{q}$. Rappelons que si $n \in \mathbb{N}^{*}$ et $q \in \mathbb{K}^{*}, E_{n}^{q}=\mathbb{K}_{q}\left[Y_{1}, Y_{2}, \ldots, Y_{n}\right]$ est l'algèbre engendrée sur $\mathbb{K}$ par $n$ générateurs $Y_{1}, \ldots, Y_{n}$ soumis aux relations $Y_{i} Y_{j}=q Y_{j} Y_{i}$, pour $1 \leq i<j \leq n$. Il est clair que $E_{n}^{q}$ est une extension de Ore itérée du corps $\mathbb{K}$, intègre et noethérienne.

Dans la première sous-section, nous rappelons la classification du spectre de $E_{n}^{q}$ pour $\mathbb{K}$ algébriquement clos et $q$ non racine de l'unité. Dans la seconde, nous étudions certains facteurs intègres de $E_{n}^{q}$ et montrons en particulier que, si $\mathbb{K}$ est algébriquement clos et $q$ non racine de l'unité, alors tous les quotients premiers de $E_{n}^{q}$ sont des ordres maximaux de leur corps de fractions.

\subsection{Spectre premier des espaces quantiques uniparamétrés}

On reprend les notations de [1]. On note $\mathcal{W}$ l'ensemble des parties de $\mathbb{N}_{n}$. Alors, si $\omega \in \mathcal{W}$, on note $\operatorname{spec}_{\omega} E_{n}^{q}$ l'ensemble des idéaux premiers $\mathfrak{p}$ de $E_{n}^{q}$ tels que $\mathfrak{p} \cap\left\{Y_{i}, i \in \mathbb{N}_{n}\right\}=\left\{Y_{i}, i \in \omega\right\}$, de sorte que le spectre de $E_{n}^{q}$, noté spec $E_{n}^{q}$, est réunion disjointe des $\operatorname{spec}_{\omega} E_{n}^{q}, \omega \in \mathcal{W}$.

Pour $\omega \in \mathcal{W}$, on note $J_{\omega}$ l'idéal engendré par $\left\{Y_{i}, i \in \omega\right\}$. Il est clair que c'est un idéal complètement premier. D'autre part, si $\omega \in \mathcal{W}$ est tel que $n-\operatorname{card} \omega=m$ soit impair et si $l_{1}<\ldots<l_{m}$ est la suite ordonnée des entiers de $\mathbb{N}_{n} \backslash \omega$, on montre aisément que pour tout $\lambda \in \mathbb{K}^{*}, Y_{l_{1}} Y_{l_{3}} \ldots Y_{l_{m}}-\lambda Y_{l_{2}} Y_{l_{4}} \ldots Y_{l_{m-1}}$ est normal modulo $J_{\omega}$ et on pose:

$$
\mathfrak{p}_{\omega, l}=J_{\omega}+\left(Y_{l_{1}} Y_{l_{3}} \ldots Y_{l_{m}}-\lambda Y_{l_{2}} Y_{4} \ldots Y_{l_{m-1}}\right)
$$

Il est connu que, pour tout $q \in \mathbb{K}^{*}$, les idéaux $\mathfrak{p}_{\omega, \lambda}$ sont complètement premiers (voir [14, Chap. 3, prop. 2.1.2]). D'autre part, lorsque $\mathbb{K}$ est algébriquement clos et $q$ non racine de l'unité, la proposition suivante classifie le spectre premier de $E_{n}^{q}$ (voir $[16, \S 3$ p. 144] et $[1,4.9])$. On a: 
Propostion 3.1.1. Supposons $\mathbb{K}$ algébriquement clos; si $q \in \mathbb{K}^{*}$ est non racine de l'unité, alors $\operatorname{spec} E_{n}^{q}=\cup_{\omega \in \mathcal{W}} \operatorname{spec}_{\omega} E_{n}^{q}$ et

si $n$ - card $\omega$ est pair, alors $\operatorname{spec}_{\omega} E_{n}^{q}=\left\{J_{\omega}\right\}$,

si $n-\operatorname{card} \omega$ est impair, alors $\operatorname{spec}_{\omega} E_{n}^{q}=\left\{J_{\omega}\right\} \cup\left\{\mathfrak{p}_{\omega, \lambda}, \lambda \in \mathbb{K}^{*}\right\}$.

\subsection{Facteurs premiers des espaces quantiques uniparamétrés}

Nous prouvons, à présent, que les facteurs premiers de $E_{n}^{q}$ par les $J_{\omega}$ et les $\mathfrak{p}_{\omega, \lambda}$ sont des ordres maximaux de leur corps de fractions. Pour les $J_{\omega}$ c'est immédiat puisque $E_{n}^{q} / J_{\omega} \cong E_{m}^{q}$, où $m=n-\operatorname{card} \omega$ (voir 1.3). Nous nous limitons donc aux $\mathfrak{p}_{\omega, \lambda}$. De plus, il est clair que pour tout idéal $\mathfrak{p}_{\omega, \lambda}$, il existe un automorphisme de $E_{n}^{q}$ qui échange $\mathfrak{p}_{\omega, 1}$ et $\mathfrak{p}_{\omega, \lambda}$. C'est pourquoi dans la suite nous n'étudierons que $\mathfrak{p}_{\omega, 1}$. Enfin, on peut se ramener au cas où $\omega=\emptyset$ en travaillant modulo $J_{\omega}$. En effet, dans les notations précédentes:

$$
E_{n}^{q} / \mathfrak{p}_{\omega, 1} \cong \mathbb{K}_{q}\left[Y_{l_{1}}, \ldots, Y_{l_{m}}\right] /\left(Y_{l_{1}} Y_{l_{3}} \ldots Y_{l_{m}}-Y_{l_{2}} Y_{l_{4}} \ldots Y_{l_{m-1}}\right)
$$

Posant $m=2 p+1$, il s'agit donc de montrer que le quotient de $E_{2 p+1}^{q}$ par l'idéal complètement premier $\left(Y_{1} Y_{3} \ldots Y_{2 p+1}-Y_{2} Y_{4} \ldots Y_{2 p}\right)$ est un ordre maximal de son corps de fractions. Pour toute la fin de cette sous-section, nous posons $Y=Y_{1} Y_{3} \ldots Y_{2 p+1}-Y_{2} Y_{4} \ldots Y_{2 p}$.

Le cas $p=0$ est trivial. Le cas $p=1$ est immédiat puisque, $E_{3}^{q} /(Y) \cong \mathbb{K}_{q}\left[Y_{1}, Y_{3}\right]$ est une extension de Ore itérée de $\mathbb{K}$ (voir 1.3).

Notons $y_{i}$ la classe de $Y_{i}$ modulo $(Y)$. Il est clair que $y_{1} y_{3} \ldots y_{2 p-1}$ est un élément normal régulier de $E_{2 p+1}^{q} /(Y)$ et que $\left(E_{2 p+1}^{q} /(Y)\right)_{y_{1} y_{3} \ldots y_{2 p-1}} \cong\left(E_{2 p+1}^{q}\right)_{Y_{1} Y_{3} \ldots Y_{2 n-1}} /(Y)$. Enfin, posant $F=\mathbb{K}_{q}\left[Y_{1}, Y_{2}, \ldots, Y_{2 p}\right]_{Y_{1} Y_{3} \ldots Y_{2 p-1}}$, on a aisément $F \cong\left(E_{2 p+1}^{q}\right)_{Y_{1} Y_{3} \ldots Y_{2 p-1}} /(Y)$. Finalement:

$$
E_{2 p+1}^{q} /(Y) \hookrightarrow\left(E_{2 p+1}^{q} /(Y)\right)_{y_{1} y_{3} \ldots y_{2,-1}} \cong F .
$$

Nous établissons à présent un lemme sur les espaces quantiques multiparamétrés utile dans la preuve de 3.2.2. Rappelons qu'un anneau est dit réduit lorsqu'il ne contient pas d'éléments nilpotents non nuls; il est connu qu'un tel anneau est semipremier.

Nous notons $\mathcal{M}_{n}^{\prime}\left(\mathbb{K}^{*}\right)$ l'ensemble des matrices $\Lambda=\left(\lambda_{i j}\right)_{1 \leq i, j \leq n}$ à coefficients dans $\mathbb{K}^{*}$ telles que $\lambda_{i i}=1$ et $\lambda_{i j}=\lambda_{j i}^{-1}$, pour $1 \leq i, j \leq n$. A une matrice $\Lambda=\left(\lambda_{i j}\right) \in \mathcal{M}_{n}^{\prime}\left(\mathbb{K}^{*}\right)$ on associe l'espace quantique multiparamétré, noté $E_{n}^{\wedge}$. C'est l'algèbre engendrée sur $\mathbb{K}$ par $n$ générateurs $Y_{1}, \ldots, Y_{n}$ soumis aux relations $Y_{i} Y_{j}=\lambda_{i j} Y_{j} Y_{i}$ pour $1 \leq i, j \leq n$. On a alors:

Lemme 3.2.1. Soient $n$ un entier tel que $n \geq 2, \Lambda=\left(\lambda_{i j}\right) \in \mathcal{M}_{n}^{\prime}\left(\mathbb{K}^{*}\right)$ et $E_{n}^{\Lambda}$ l'espace quantique multiparamétré associé. On considère deux entiers $r$ et $s$ tels que $1 \leq r<s \leq n$ et on pose $m=Y_{1} \ldots Y_{r}$ et $m^{\prime}=Y_{r+1} \ldots Y_{s}$. Alors, l'anneau quotient $E_{n}^{\wedge} /\left(m, m^{\prime}\right)$ est réduit.

Preuve. On a $E_{n}^{\wedge}=\mathbb{K}\left[Y_{1}\right]\left[Y_{2} ; \sigma_{2}\right] \ldots\left[Y_{n} ; \sigma_{n}\right]$, où pour $2 \leq i \leq n, \sigma_{i}$ est l'automorphisme 
de $\mathbb{K}\left[Y_{1}\right]\left[Y_{2} ; \sigma_{2}\right] \ldots\left[Y_{i-1} ; \sigma_{i-1}\right]$ défini, pour $1 \leq j \leq i-1$, par $\sigma_{i}\left(Y_{j}\right)=\lambda_{i j} Y_{j}$. Comme l'idéal $\left(Y_{1} \ldots Y_{r}\right)$ de $\mathbb{K}\left[Y_{1}\right]\left[Y_{2} ; \sigma_{2}\right] \ldots\left[Y_{r} ; \sigma_{r}\right]$ est $\sigma_{i}$-stable pour $r+1 \leq i \leq n$, il vient:

$$
E_{n}^{\Lambda} /\left(m, m^{\prime}\right) \cong\left(A\left[Y_{r+1} ; \bar{\sigma}_{r+1}\right] \ldots\left[Y_{n} ; \bar{\sigma}_{n}\right]\right) /\left(Y_{r+1} \ldots Y_{s}\right),
$$

où $A=\mathbb{K}\left[Y_{1}\right]\left[Y_{2} ; \sigma_{2}\right] \ldots\left[Y_{r} ; \sigma_{r}\right] /\left(Y_{1} \ldots Y_{r}\right)$ et où, pour $r+1 \leq i \leq n, \bar{\sigma}_{i}$ est l'automorphisme induit sur $A$ par $\sigma_{i}$.

Posons $B=A\left[Y_{r+1} ; \bar{\sigma}_{r+1}\right] \ldots\left[Y_{n} ; \bar{\sigma}_{n}\right]$; comme $B$ est un $A$-module libre à gauche de base $\left\{Y_{r+1}^{j_{r+1}} \ldots Y_{n}^{j_{n}},\left(j_{r+1}, \ldots, j_{n}\right) \in \mathbb{N}^{n-r}\right\}$, on a $\left(Y_{r+1}\right) \cap\left(Y_{r+2}\right) \cap \ldots \cap\left(Y_{s}\right)=\left(Y_{r+1} \ldots Y_{s}\right)$ dans $B$. Considérons alors l'application $\phi: B \rightarrow B /\left(Y_{r+1}\right) \times \ldots \times B /\left(Y_{s}\right)$ définie par $\phi(f)=$ $\left(f+\left(Y_{r+1}\right), \ldots, f+\left(Y_{s}\right)\right)$. D'après ce qui précède, $\operatorname{ker} \phi=\left(Y_{r+1} \ldots Y_{s}\right)$. On dispose donc d'une injection:

$$
E_{n}^{\wedge} /\left(m, m^{\prime}\right) \cong B /\left(Y_{r+1} \ldots Y_{s}\right) \hookrightarrow B /\left(Y_{r+1}\right) \times \ldots \times B /\left(Y_{s}\right) .
$$

D'autre part, pour $r+1 \leq i \leq s$, on a $B /\left(Y_{i}\right) \cong E_{n}^{\wedge} /\left(Y_{1} \ldots Y_{r}, Y_{i}\right) \cong E_{i} /\left(Y_{1} \ldots Y_{r}\right)$, où $E_{i}$ désigne le sous-anneau de $E_{n}^{\Lambda}$ engendré par le $Y_{j}$ pour $j \neq i$. Il est clair que $E_{i}$ est un espace quantique multiparamétré et que, dans $E_{i}$, l'idéal $\left(Y_{1} \ldots Y_{r}\right)$ est intersection d'idéaux complètement premiers: $\left(Y_{1} \ldots Y_{r}\right)=\left(Y_{1}\right) \cap \ldots \cap\left(Y_{r}\right)$. Ainsi, pour $r+1 \leq i \leq s$, $B /\left(Y_{i}\right)$ est un anneau réduit et par suite $B /\left(Y_{r+1}\right) \times \ldots \times B /\left(Y_{s}\right)$ est un anneau réduit. Il s'ensuit que $E_{n}^{\wedge} /\left(m, m^{\prime}\right)$ est réduit.

Proposition 3.2.2. Soit $\mathcal{I}$ (resp. $\mathcal{J}$ ) l'ensemble des entiers impairs (resp. pairs) de $\mathbb{N}_{2 p}$. Pour $(i, j) \in \mathcal{I} \times \mathcal{J}$, on pose $\mathcal{P}_{i, j}=\left(y_{i}, y_{j}\right) \triangleleft E_{2 p+1}^{q} /(Y)$. Alors:

(i) pour tout $(i, j) \in \mathcal{I} \times \mathcal{J}, \mathcal{P}_{i, j}$ est complètement premier,

(ii) $\left\{\mathcal{P}_{i, j},(i, j) \in \mathcal{I} \times \mathcal{J}\right\}$ est l'ensemble des idéaux premiers minimaux au dessus de $\left(y_{1} y_{3} \ldots y_{2 p-1}\right)$,

(iii) $\left(y_{1} y_{3} \ldots y_{2 p-1}\right)=\cap_{I \times \mathcal{J}} \mathcal{P}_{i, j}$.

Preuve. Pour $(i, j) \in \mathcal{I} \times \mathcal{J}$, l'idéal $\left(Y_{i}, Y_{j}\right)$ de $E_{2 p+1}^{q}$ est complètement premier et contient $(Y)$. On en déduit (i). D'autre part, soit $\mathcal{P}$ un idéal premier minimal au dessus de $\left(y_{1} y_{3} \ldots y_{2 p-1}\right)$. Alors, $y_{1} y_{3} \ldots y_{2 p-1}$ et $y_{2} y_{4} \ldots y_{2 p}$ sont dans $\mathcal{P}$. Puisque, pour $k \in \mathbb{N}_{2 p+1}$, les $y_{k}$ sont normaux, il existe donc $(i, j) \in \mathcal{I} \times \mathcal{J}$ tel que $\left(y_{i}, y_{j}\right) \subseteq \mathcal{P}$. D'après (i) on a alors $\mathcal{P}=\mathcal{P}_{i, j}$ d'où (ii). Enfin, remarquons que $\left(E_{2 p+1}^{q} /(Y)\right) /\left(y_{1} y_{3} \ldots y_{2 p-1}\right) \cong$ $E_{2 p+1}^{q} /\left(Y_{1} Y_{3} \ldots Y_{2 p-1}, Y_{2} Y_{4} \ldots Y_{2 p}\right)$. Quitte à réindexer les $Y_{k}$, le lemme 3.2.1 s'applique à $E_{2 p+1}^{q} /\left(Y_{1} Y_{3} \ldots Y_{2 p-1}, Y_{2} Y_{4} \ldots Y_{2 p}\right)$. On en déduit que $\left(E_{2 p+1}^{q} /(Y)\right) /\left(y_{1} y_{3} \ldots y_{2 p-1}\right)$ est semipremier, d'où (iii).

Corollaire 3.2.3. L'anneau intègre $E_{2 p+1}^{q} /(Y)$ est un ordre maximal de son corps de fractions.

Preuve. D'après 3.2.2, l'idéal $\left(y_{1} y_{3} \ldots y_{2 p-1}\right)$ de $E_{2 p+1}^{q} /(Y)$ est intersection des idéaux 
complètement premiers $\mathcal{P}_{i, j}$ pour $(i, j) \in \mathcal{I} \times \mathcal{J}$. L'automorphisme associé à l'élément normal régulier $y_{1} y_{3} \ldots y_{2 p-1} \in E_{2 p+1}^{q} /(Y)$ agit par multiplication scalaire sur les $y_{i}$. Il laisse donc stable les idéaux $\mathcal{P}_{i, j},(i, j) \in \mathcal{I} \times \mathcal{J}$. Enfin, d'après $(9),\left(E_{2 p+1}^{q} /(Y)\right)_{y_{1} y_{3} \ldots y_{2-1}}$ est isomorphe à un localisé d'espace quantique multiparamétré; à ce titre, c'est un ordre maximal de son corps de fractions (voir 1.3). On est donc dans les hypothèses du lemme 1.1 qui permet de conclure.

D'après 3.2 .3 et 3.1 .1 , on a finalement:

Théorème 3.2.4. Soit $n \in \mathbb{N}^{*}$ et $q \in \mathbb{K}^{*}$. Dans les notations de 3.1,

(i) si $(\omega, \lambda) \in \mathcal{W} \times \mathbb{K}^{*}$, alors $E_{n}^{q} / J_{\omega}$ et $E_{n}^{q} / \mathfrak{p}_{\omega, \lambda}$ sont des anneaux intègres et des ordres maximaux de leur corps de fractions;

(ii) en particulier, si $\mathbb{K}$ est algébriquement clos et q non racine de l'unité, alors tout facteur (complètement) premier de $E_{n}^{q}$ est un ordre maximal de son corps de fractions.

La remarque suivante énonce sans preuve certains résultats de factorialité concernant $E_{n}^{q}$ et ses quotients premiers. Les preuves de ces résultats se trouvent dans [14, Chap. $3 \$ 1.1$ et 2.2].

Remarque 3.2.5. La notion d'anneau noethérien factoriel (non nécéssairement commutatif) est introduite dans [5]. Un anneau $R$ est dit noethérien factoriel si il est noethérien premier et si tout idéal premier non nul contient un idéal principal (c'est-àdire engendré par un élément normal) premier et non nul. D'après [5, 2.4], un anneau noethérien intègre factoriel est un ordre maximal de son corps de fractions. A l'aide de $[5,4.2]$ on montre que tout espace quantique multiparamétré $E_{n}^{\wedge}$ est un anneau noethérien factoriel $([14$, Chap. 3 cor. 1.2.5]). Cependant, dans les notations précédentes, on peut montrer que pour $p \geq 2, E_{2 p+1}^{q} /(Y)$ n'est pas un anneau factoriel ([14, Chap. 3 cor. 2.2.6]). En particulier, il n'est pas isomorphe à un espace quantique multiparamétré. Ainsi, pour $(\omega, \lambda) \in \mathcal{W} \times \mathbb{K}^{*}$ tel que $n-$ card $\omega$ soit impair, si $n-\operatorname{card} \omega \neq 1$ ou $3, E_{n}^{q} / \mathfrak{p}_{\omega, \lambda}$ est un ordre maximal de son corps de fractions sans être un anneau factoriel.

Nous terminons par une remarque concernant certains facteurs complètement premiers de l'algèbre de Weyl quantique $A_{n}^{\bar{q}, \Lambda}$.

Remarque 3.2.6. (1) On reprend les définitions et notations de [13]. Soit $n \in \mathbb{N}^{*}$; à $\bar{q}=\left(q_{1}, \ldots, q_{n}\right) \in\left(\mathbb{K}^{*}\right)^{n}$ et $\Lambda \in \mathcal{M}_{n}^{\prime}\left(\mathbb{K}^{*}\right)$, on associe l'algèbre $A_{n}^{\bar{q}, \Lambda}$ définie au 2.1.1 de [13]. Nous supposons les $q_{i}$ distincts de 1 , disposant ainsi des résultats de [13, §3.1]. Pour la définition d'idéal algorithmique de $A_{n}^{\bar{q}, \Lambda}$, nous renvoyons à [13, §3.1]. Rappelons que ces idéaux sont complètement premiers $([13,3.1 .6])$. En appliquant les méthodes développées dans le présent travail, on montre que si $P$ est un idéal algorithmique de $A_{n}^{\bar{q}, \Lambda}$, alors l'anneau intègre $A_{n}^{\bar{q} \wedge} / P$ est un ordre maximal de son corps de fractions ([14, Chap. 3 théo. 3.4]). 
(2) De plus, d'après $[13,3.2 .3]$, si $\mathbb{K}$ est algébriquement clos et de caractéristique nulle et si le sous-groupe de $\mathbb{K}^{*}$ engendré par les coefficients de $\bar{q}$ et $\Lambda$ est libre de rang $\frac{1}{2} n(n+1)$, alors le spectre de $A_{n}^{\dot{q}, \Lambda}$ est réduit aux idéaux maximaux (dont les anneaux résiduels sont isomorphes à $\mathbb{K}$ ) et aux idéaux algorithmiques. En fait, sous ces hypothèses, et en utilisant la famille complète de générateurs d'un idéal algorithmique (définie en $[13,3.1 .8]$ ), on montre que tous les facteurs premiers de $A_{n}^{\bar{q}, \Lambda}$ sont factoriels.

\section{RÉFÉRENCES}

1. K. A. Brown and K. R. Goodearl, Prime spectra of quantum semisimple groups, Trans. Amer. Math. Soc. 348 (1996), 2465-2502.

2. W. Bruns and U. VetTer, Determinantal rings (Lecture Notes in Math. 1327, SpringerVerlag, Berlin, 1988).

3. M. Chamarie, Localisations dans les ordres maximaux, Comm. Algebra 2 (1974), 279-293.

4. M. Chamarie, Sur les ordres maximaux au sens d'Asano (Vorlesungen aus dem Fachbereich Mathematik der Universität Essen, Heft 3, 1979).

5. A. W. Chatters and D. A. Jordan, Noncommutative unique factorisation rings, $J$. London Math. Soc. (2) 33 (1986), 22-32.

6. K. R. Goodearl, Prime ideals in skew polynomial rings and quantized Weyl algebras, $J$. Algebra 150 (1992), 324-377.

7. K. R. Goodearl and T. H. Lenagan, Quantum determinantal ideals, preprint.

8. G. MaUry, Ordres maximaux preque-factoriels, Comm. Algebra 8 (1980), 1711-1720.

9. G. Maury et J. Raynaud, Ordres maximaux au sens de $K$. Asano (Lecture Notes in Math. 808, Springer-Verlag, Berlin, 1980).

10. J. C. MCCONNELl and J. C. Robson, Noncommutative Noetherian Rings (Wiley, Chichester, 1987).

11. M. Noumi, H. Yamada and K. Mimachi, Finite dimensional representations of the quantum group $G L_{q}(n ; C)$ and the zonal spherical functions on $U_{q}(n-1) \backslash U_{q}(n)$, Japan. J. Math. 19 (1993), 31-80.

12. B. Parshall and J. Wang, Quantum Linear Groups, Mem. Amer. Math. Soc. 89 (1991), no. 439.

13. L. Rigal, Spectre de l'algèbre de Weyl quantique, Beiträge Algebra Geom. 37 (1996), 119-148.

14. L. Rigal, Analogues quantiques de l'Algèbre de Weyl et Ordres Maximaux quantiques (Thèse de Doctorat de l'Université Paris VI, 1996).

15. D. W. Sharpe, On certain polynomial ideals defined by matrices, Quart. J. Math. Oxford Ser. (2) 15 (1964), 155-175. 
16. S. P. Smith, Quantum groups: an introduction and survey for ring theorists, in Noncommutative Rings (Berkeley, CA, 1989), (Math. Sci. Res. Inst. Publ. 24, Springer-Verlag, New York, 1992), 131-178.

Université de Poitiers

DÉPARTEMENT DE MATHÉmatiQues 40, Avenue du Recteur Pineau 86022 POITIERS

France

\section{Current address:}

Université de SAINT-ETIENNE

Faculté des SCiences et TeChNiques, Mathématiques

23, Rue du Docteur Paul Michelon

42023 Saint-Etienne Cedex

FRANCE

E-mail address: Laurent.Rigal@univ-st-etienne.fr 